'Department of Medicine, Division of Nephrology, The Icahn School of Medicine at Mount Sinai, New York, $N Y$, USA.

${ }^{2}$ Research and Development, Prime Medicine, Cambridge, MA, USA

${ }^{3}$ Department of Medicine University of Washington, Seattle, WA, USA

${ }^{4}$ Department of Medicine, Massachusetts General Hospital, Boston, MA, USA.

$\otimes_{\text {-mail: }}$

ilse.daehn@mssm.edu: jduffield@primemedicine.com

https://doi.org/10.1038 \$41573-021-00242-0

\title{
The glomerular filtration barrier: a structural target for novel kidney therapies
}

IIse S. Daehn $\mathbb{1}^{1 凶}$ and Jeremy S. Duffield $\mathbb{B}^{2,3,4 凶}$

Abstract | Loss of normal kidney function affects more than $10 \%$ of the population and contributes to morbidity and mortality. Kidney diseases are currently treated with immunosuppressive agents, antihypertensives and diuretics with partial but limited success. Most kidney disease is characterized by breakdown of the glomerular filtration barrier (GFB). Specialized podocyte cells maintain the GFB, and structure-function experiments and studies of intercellular communication between the podocytes and other GFB cells, combined with advances from genetics and genomics, have laid the groundwork for a new generation of therapies that directly intervene at the GFB. These include inhibitors of apolipoprotein L1 (APOL1), short transient receptor potential channels (TRPCs), soluble fms-like tyrosine kinase 1 (sFLT1; also known as soluble vascular endothelial growth factor receptor 1), roundabout homologue 2 (ROBO2), endothelin receptor $\mathrm{A}$, soluble urokinase plasminogen activator surface receptor (suPAR) and substrate intermediates for coenzyme Q10 $\left(\mathrm{CoQ}_{10}\right)$. These molecular targets converge on two key components of GFB biology: mitochondrial function and the actin-myosin contractile machinery. This Review discusses therapies and developments focused on maintaining GFB integrity, and the emerging questions in this evolving field.

The breakdown of the glomerular filtration barrier (GFB) manifests as a protein leak from the plasma into the urine (proteinuria), is associated with loss of normal kidney function and is a powerful risk factor for disease progression to organ failure ${ }^{1}$. Clinically, patients may present with nephrotic syndrome (a high-grade form of proteinuria with oedema), isolated proteinuria or proteinuria with hypertension. Abnormal kidney function may also be detected upon blood testing. Childhood nephrotic syndrome is managed by clinical assessment and is determined to be sensitive to steroid therapy or steroid-resistant nephrotic syndrome (SRNS). In adults, GFB disease is frequently named according to the pathological lesions detected histologically following a kidney biopsy. The classical adult pathological descriptors of GFB disease are minimal change disease and focal segmental glomerulosclerosis (FSGS). However, common and rare diseases that present with protein leak into the urine, including hypertensive nephroangiosclerosis, diabetic kidney disease, lupus nephritis, pre-eclampsia and Alport syndrome, frequently have GFB disease as a major component upon histological inspection (TABLE 1). GFB breakdown results in glomerular cell activation, local scar tissue formation, occlusion of glomerular capillaries, impaired blood flow to the nephron and, ultimately, glomerular obsolescence. In addition, plasma proteins that leak into the glomerular filtrate directly injure the tubular epithelium, resulting in loss of tubular function and interstitial scarring ${ }^{2-4}$. Therefore, the burden of GFB disease on human health is enormous. Occasionally, patients have reduced kidney function without damage to the GFB. In these infrequent cases, patients do not have proteinuria, and inspection of the kidney indicates that damage to the tubules and their supportive capillaries underlies the kidney disease.

Existing therapies for kidney disease have largely treated complications, symptoms or late manifestations of disease, and have had limited disease-modifying effects. Encouragingly, in recent trials, sodium-glucose co-transporter 2 (SGLT2) inhibitors have improved hyperglycaemia control as well as cardiovascular and renal outcomes in patients with advanced type 2 diabetes mellitus ${ }^{5,6}$. However, despite their recognized pleiotropic effects on glomerular cells ${ }^{7,8}$, SGLT2 inhibitors act predominantly on the kidney tubule and have little direct role in GFB function, and thus have only minor effects on proteinuria ${ }^{5,9}$.

Recent large epidemiological studies have demonstrated an important predictive link between proteinuria and disease progression to organ failure in both 
Table 1 | Kidney diseases in which the GFB is implicated in pathogenesis

\begin{tabular}{|c|c|c|}
\hline Disease & Clinical manifestations & Potential molecular targets \\
\hline Minimal change disease & Nephrotic syndrome & $\begin{array}{l}\text { APOL1, TRPC6, TRPC5, suPAR, ET } \mathrm{A} \text {, } \\
\mathrm{CoQ}_{10} \text { biosynthesis }\end{array}$ \\
\hline Focal segmental glomerulosclerosis & $\begin{array}{l}\text { Nephrotic syndrome, proteinuria, } \\
\text { hypertension, CKD }\end{array}$ & $\begin{array}{l}\text { APOL1, TRPC6, TRPC5, suPAR, ET } \text { A }^{\text {R }} \\
\text { CoQ }_{10} \text { biosynthesis }\end{array}$ \\
\hline Alport syndrome & $\begin{array}{l}\text { Nephrotic syndrome, proteinuria, } \\
\text { haematuria, hypertension, CKD }\end{array}$ & miR-21, NRF2 \\
\hline $\begin{array}{l}\text { Diabetic kidney disease (GFB partially } \\
\text { implicated) }\end{array}$ & $\begin{array}{l}\text { Nephrotic syndrome, proteinuria, } \\
\text { hypertension, CKD }\end{array}$ & TRPC5, TRPC6, ET ${ }_{A} R$, miR-21 \\
\hline $\begin{array}{l}\text { Hypertensive nephroangiosclerosis } \\
\text { (GFB partially implicated) }\end{array}$ & Proteinuria, hypertension, CKD & APOL1, TRPC6, ET ${ }_{A} R$ \\
\hline Pre-eclampsia & Nephrotic syndrome, hypertension & sFLT1 \\
\hline Lupus nephritis & $\begin{array}{l}\text { Nephrotic syndrome, proteinuria, } \\
\text { hypertension, CKD }\end{array}$ & APOL1, ET $T_{A} R$ \\
\hline Other immune complex disease & $\begin{array}{l}\text { Nephrotic syndrome, proteinuria, } \\
\text { hypertension, CKD }\end{array}$ & $\mathrm{ET}_{\mathrm{A}} \mathrm{R}$, complement \\
\hline \multicolumn{3}{|c|}{$\begin{array}{l}\text { APOL1, apolipoprotein } \mathrm{L1} \text {; CKD, chronic kidney disease; } \mathrm{CoQ}_{10} \text {, coenzyme } \mathrm{Q} 10 ; \mathrm{GFB} \text {, glomerular filtration barrier; } \mathrm{ET}_{\mathrm{A}} \mathrm{R} \text {, endothelin } \\
\text { type A receptor; miR-21, microRNA-21; NRF2, nuclear factor erythroid 2-related factor 2; sFLT1, soluble fms-like tyrosine kinase } 1 \\
\text { (also known as soluble vascular endothelial growth factor receptor 1); suPAR, soluble urokinase plasminogen activator surface } \\
\text { receptor; TRPC, transient receptor potential channel. }\end{array}$} \\
\hline
\end{tabular}

common and rare diseases ${ }^{10}$. These observations have prompted regulatory organizations to recommend proteinuria as an approvable primary end point in settings where protein levels in the urine are high. This simpler primary end point will likely enable the evaluation of investigational therapies in patients and accelerate the drug discovery process. These welcome changes coincide with new insights into the mechanisms by which kidneys spill protein into the urine, following 20 years of research on the GFB. In this Review, we provide an overview of the GFB, introduce novel candidate therapies that specifically target the key aspects of GFB function and discuss challenges and potential opportunities for future research and development (TABLE 2).

\section{Overview of the GFB}

The GFB (FIG. 1) was first described more than 50 years ago, and is a unique structure comprising highly fenestrated and specialized endothelial cells, an unusual basement membrane and a high density of arborized interdigitating cells known as podocytes ${ }^{11}$. The GFB is the terminal unit through which the kidney filters water, salts and low molecular weight organic molecules from the blood. Podocytes form the architectural backbone and provide the mechanical stability of the $\mathrm{GFB}^{12,13}$. Because of the mechanically demanding environment, cytoskeletal integrity of podocytes is paramount for their function ${ }^{14}$. The specialized projections that interdigitate to form the slit diaphragm are key elements in the GFB and contribute to its overall physical stability ${ }^{15}$. The central molecular components of the slit diaphragm were first identified in 1998 and 2000, when the genes that cause childhood nephrotic syndrome, NPHS1 (which encodes nephrin) and NPHS2 (which encodes podocin), were discovered and found to be exclusively expressed at the slit diaphragm ${ }^{16,17}$. These proteins anchor the cytoskeleton to the plasma membrane. Nephrin (also known as NEPHS1), along with similar proteins such as FAT1 and NEPH1/2, form a multilayered bipartite scaffold between each of the interdigitating podocyte projections (tertiary foot processes) ${ }^{18}$. The proteins form flexible spring-like protein bridges that prevent macromolecules from passing through. Since the discovery of these key proteins, an array of molecular components that maintain the podocyte tertiary foot processes through dynamic regulation of the actin cytoskeleton (such as those encoded by ACTN4, INF2, ARHGAP24, MYH9 and TRPC6) have been associated with the development of FSGS and nephrotic syndrome ${ }^{19-23}$. Additional proteins that maintain slit diaphragm proteins (for example, CD2-associated protein (CD2AP)) as well as proteins that tether the podocyte to the basement membrane (such as $\alpha 3 \beta 2$ integrin) all play vital roles in GFB maintenance.

One of the earliest structural features of GFB dysfunction is the retraction of podocyte tertiary foot processes, which reduces the podocyte surface area and disrupts the slit diaphragm ${ }^{24}$. Persistent podocyte dysfunction leads to deposition of abnormal basement membrane matrix, which makes the tissue sclerotic and, ultimately, results in podocyte depletion ${ }^{25}$. Recent ultrastructural analyses indicate that alterations in the number and the morphology of foot processes and/or shortening of the slit diaphragm in experimental FSGS leads to capillary dilation due to reduced compressive forces that counteract filtration pressure, which contributes to GFB breakdown and, ultimately, results in protein leak ${ }^{26}$.

Podocytes regulate glomerular endothelial cell (GEC) growth, survival, differentiation and permeability by releasing the essential paracrine cytokine, vascular endothelial growth factor A (VEGFA), which binds to its cognate receptor, VEGFR2, on GECs ${ }^{27,28}$. GECs are highly specialized cells with fenestrae and a charged luminal endothelial surface layer also known as the 'glycocalyx', which is a negatively charged network of proteoglycans, glycoproteins and glycolipids ${ }^{29-31}$ that helps maintain a charge-selective barrier ${ }^{32,33}$. GEC dysfunction can also initiate and contribute to GFB breakdown $n^{11,31,34}$. 
Table 2 | Summary of ongoing clinical trials for drugs targeting the GFB

\begin{tabular}{|c|c|c|c|c|c|}
\hline Drug (company) & Study population & Target & Type of molecule & Status & $\begin{array}{l}\text { Refs and NCT } \\
\text { identifier }\end{array}$ \\
\hline $\begin{array}{l}\text { Bardoxolone methyl } \\
\text { (Reata Pharmaceuticals) }\end{array}$ & Alport syndrome & NRF2 & Small molecule & Phase II/III & $\begin{array}{l}\text { NCT03019185 } \\
\left.\text { (REF. }^{65}\right)\end{array}$ \\
\hline VAR-200 (ZyVersa) & FSGS & Cholesterol efflux & Small molecule & Phase I & 69,221 \\
\hline $\begin{array}{l}\text { ION532 (lonis Pharmaceuticals/ } \\
\text { AstraZeneca) }\end{array}$ & FSGS & APOL1 & Antisense oligonucleotide & Phase I & 95 \\
\hline AM-1473 (Amgen) & FSGS & TRPC6 & Small molecule & Preclinical & 130 \\
\hline GFB-887 (Goldfinch Bio) & FSGS, DKD, TR-MCD & TRPC5 & Small molecule & Phase lla & NCT04387448 \\
\hline Antibody (Walden Biosciences) & FSGS & suPAR & Neutralizing antibody & Preclinical & 145 \\
\hline Atrasentan (Chinook Therapeutics) & $\begin{array}{l}\text { FSGS, DKD, IgAN, } \\
\text { Alport syndrome }\end{array}$ & $\mathrm{ET}_{\mathrm{A}}$ & Small molecule & Phase III & NCT04573920 \\
\hline Sparsentan (Travere Therapeutics) & $\lg A N$ & $\mathrm{ARB}$ and $\mathrm{ET}_{\mathrm{A}}$ & Small molecule & Phase III & $\begin{array}{l}\text { NCT03762850 } \\
\left.\text { (REF. }{ }^{222}\right)\end{array}$ \\
\hline $\begin{array}{l}\text { siRNA (RNA Therapeutics Institute, } \\
\text { University of Massachusetts) }\end{array}$ & Pre-eclampsia & sFLT1 & siRNA & Late preclinical & 204 \\
\hline Apheresis (Miltenyi Biotec) & Pre-eclampsia & sFLT1 & Apheresis with TheraSorb & Phase $0 a-0 b$ & NCT02923206 \\
\hline $\begin{array}{l}\text { Apheresis (Massachusetts General } \\
\text { Hospital, Kaneka) }\end{array}$ & Pre-eclampsia & sFLT1 & Apheresis & Phase lb & $\begin{array}{l}\text { NCT01404910 } \\
\text { completed }\end{array}$ \\
\hline \multicolumn{6}{|c|}{$\begin{array}{l}\text { APOL1, apolipoprotein L1; ARB, angiotensin receptor blocker; DKD, diabetic kidney disease; } \mathrm{ET}_{\mathrm{A}} \text {, endothelin type A; Fc, crystallizable fragment; FSGS, focal } \\
\text { segmental glomerulosclerosis; GFB, glomerular filtration barrier; IgAN, IgA nephropathy; miR-21, microRNA-21; NRF2, nuclear factor erythroid 2-related factor 2; } \\
\text { ROBO2, roundabout homologue 2; sFLT1, soluble fms-like tyrosine kinase } 1 \text { (also known as soluble vascular endothelial growth factor receptor 1); siRNA, small } \\
\text { interfering RNA; suPAR, soluble urokinase plasminogen activator surface receptor; TR-MCD, treatment-resistant minimal change disease; TRPC, transient receptor } \\
\text { potential channel. }\end{array}$} \\
\hline
\end{tabular}

Some human kidney diseases resulting in GFB breakdown, such as pre-eclampsia and haemolytic uraemic syndrome, are caused by factors directly targeting the glomerular endothelium ${ }^{35}$. Human genetic association studies, combined with models of podocyte injury in gene-targeted animals, suggest that endothelial-specific nitric oxide synthetase (eNOS) plays a role in disease susceptibility and severity ${ }^{36-43}$. Furthermore, abnormalities of the components of the glomerular basement membrane (GBM), such as mutations and variants in collagen type IV basement membrane proteins that are found in common and rare diseases ${ }^{44-46}$, revealed the essential role of GBM constituents for the integrity of the GFB. The emerging picture is that podocytes, GECs and the GBM all contribute to the overall structure and function of this complex barrier.

Genetic studies of patients with familial and sporadic forms of rare kidney diseases have provided variants and mutations in many disease-causing proteins that are highly expressed by podocytes, and, less frequently, highly expressed by GECs. Emerging whole-exome sequencing and genome-wide studies indicate that these same genes are abnormally expressed or harbour mutations in a significant proportion of patients presenting with common forms of kidney disease ${ }^{47}$. Nevertheless, GFB breakdown for many is caused predominantly by environmental factors including xenobiotics, infections, local immune protein activation or immune cell activation, local metabolic disturbance from a diabetic environment or elevated glomerular pressure.

As our understanding of the GFB has evolved during the past 20 years, cell biology, comparative medicine and genetic experiments from humans have pointed to podocytes as critical to maintaining GFB function, and shown that podocyte injury or death causes scarring lesions in the glomerulus. A number of genes that are mutated or contain variants that confer increased disease risk are expressed by podocytes. Several of these have emerged as potential therapeutic targets. Circulating proteins that contribute to GFB breakdown following kidney transplantation may also be targeted for therapeutic benefit. Such proteins and factors can stimulate an injury response in podocytes, although the mechanisms by which they cause injury are not uniformly understood. Circulating complement proteins, immune complexes or autoantibodies are known to cause glomerular cell injury, in part through receptor-mediated interactions. Other circulating proteins and factors may similarly cause cell injury through receptor-ligand interactions.

The majority of pathological mutations in genes identified to cause isolated FSGS or SRNS are reported to regulate the assembly, turnover and degradation of the actin cytoskeleton or regulate its attachment at the cell surface $^{46,48}$, and other mutations cluster in the proteins 
that regulate the biosynthesis of mitochondrial constituents (TABLE 2). The following sections cover candidate therapies in development that directly target the GFB and have advanced to clinical trials or are at the late preclinical stage of development. Many of these candidate therapies impinge directly on functions of mitochondria or the actin-myosin contractile machinery, which are central to GFB function.
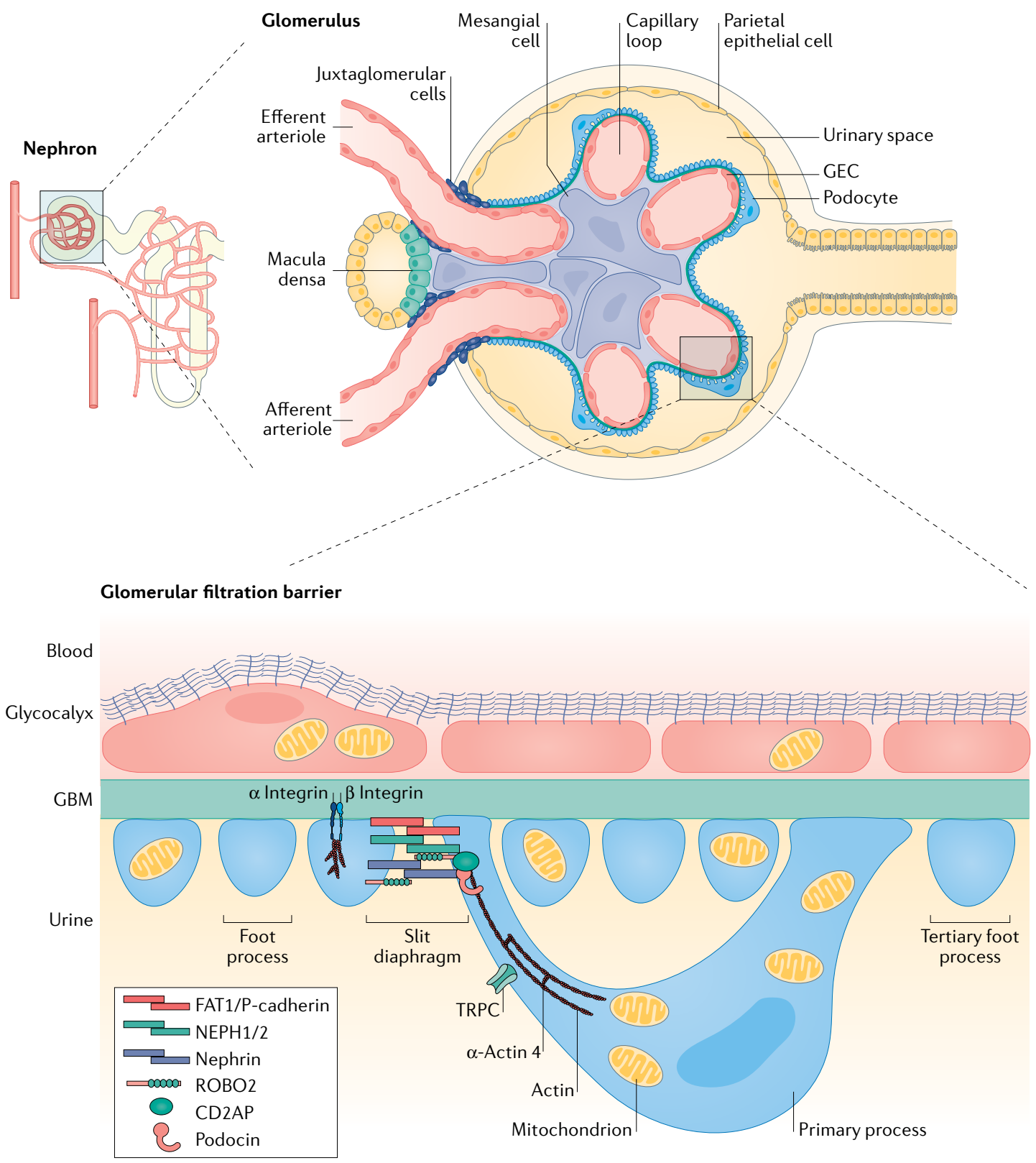

Fig. 1 Introduction to the GFB. Sequentially magnified schematic showing the nephron, the functional unit of the kidney, followed by the glomerulus, the primary interface between the microvascular circulation and the nephron. Each glomerular capillary loop forms a glomerular filtration barrier (GFB) comprising the glycocalyx and fenestrated glomerular endothelial cells (GECs), which block negatively charged molecules and plasma proteins; the glomerular basement membrane (GBM), which provides a physical scaffold that supports the GECs and podocytes, but contributes little directly to GFB function; and podocytes, which form primary, secondary and tertiary (foot) processes. Foot processes attach to the GBM by matrix tethering receptors including dystroglycans and integrins that, in turn, regulate the actin-myosin contractile apparatus to maintain foot process projections. Primary and secondary processes have an elaborate actinmyosin contractile apparatus and cross-link proteins running inside foot processes. The slit diaphragm, an ultrastructural molecular barrier, connects interdigitating foot processes, and contains NEPH1/2, P-cadherin, protocadherin FAT1, nephrin and roundabout homologue 2 (ROBO2). This molecular bridge forms part of the GFB and connects with the actin-myosin contractile apparatus via CD2-associated protein (CD2AP) and podocin. External short transient receptor potential channels (TRPCs) open in response to diverse stimuli, including mechanical forces, to release $\mathrm{Ca}^{2+}$ and regulate cellular responses. The function and localization of mitochondria in both podocytes and GECs, particularly in tertiary processes, is important in the maintenance of the GFB. Adapted from REF. ${ }^{70}$. 


\section{Targeting mitochondrial function}

Mitochondria are critical to cellular metabolism, homeostasis and stress responses, and their dysfunction is linked to diseases of the GFB. Mitochondria have roles in the pathological generation of excessive reactive oxygen species (ROS), the regulation of intracellular calcium levels, cell death pathways and cytoskeletal dynamics ${ }^{49-51}$. In this section, we discuss genetic variants in mitochondrial proteins that disrupt mitochondrial electron transport or biogenesis and contribute to GFB disruption. Although the precise contribution of mitochondria to oxidative phosphorylation in podocytes and GECs is not fully elucidated ${ }^{52,53}$, mitochondria produce ROS, control cell survival by maintaining the mitochondrial membrane potential and regulate additional cell processes including metabolism, protein translation, iron homeostasis and DNA repair.

Abnormalities of podocyte function have been linked to mitochondrial disorders ${ }^{54-56}$. Podocytes use large amounts of ATP to maintain the high surface area of tertiary foot processes via the actin cytoskeleton, and mutations in mitochondrial DNA and proteins could cause breakdown of the GFB because this ATP is lost; experimental evidence supports this hypothesis ${ }^{53,57}$. In humans, xenobiotics, including tenofovir, that inhibit mitochondrial DNA replication can cause nephrotoxicity with glomerular scarring and proteinuria, underscoring the important role for mitochondrial homeostasis in maintaining the $\mathrm{GFB}^{58}$.

Recent experimental data support additional roles for mitochondria beyond ATP production, including ROS production, cell death, integration of signalling pathways and responses to cell stress. High-content imaging studies demonstrate a relationship between mitochondrial dysfunction and changes in mitochondrial motility, fission and fusion events, localization and shape $\mathrm{e}^{59,60}$. Mitochondrial dysfunction in GECs, rather than podocytes, could also contribute to GFB loss. Mitochondrial oxidative stress lesions in GECs have been reported in patients and in experimental models of FSGS, as well as in segmental glomerular scars in patients with diabetic kidney disease ${ }^{61-63}$. Mitochondrial stress in GECs is associated with loss of fenestrations and the glycocalyx ${ }^{64}$.

Although much remains to be learned about the precise roles of mitochondria in GFB health and disease, mitochondrial function could be boosted as a broad-based approach to treat GFB disease. Several agents that may promote mitochondrial health are currently under clinical evaluation. These include a small-molecule activator of the transcription factor nuclear factor erythroid 2-related factor 2 (NRF2) from Reata Pharmaceuticals, which improved kidney function in a number of glomerular diseases, although it did not reduce protein leak at the GFB (ClinicalTrials.gov identifier NCT03019185) ${ }^{65}$. NRF2 activates both antioxidant and mitochondrial biogenesis programmes in cell ${ }^{66,67}$. Lademirsen, developed by Sanofi-Genzyme, is an antisense oligonucleotide (ASO) that inhibits microRNA-21 (miR-21), has been reported to enhance mitochondrial function in podocytes and is under evaluation in patients with Alport syndrome (NCT02855268) ${ }^{68}$. ZyVersa is planning to evaluate VAR-200 (2-hydroxypropyl- $\beta$-cyclodextrin) in patients with FSGS. VAR-200 may upregulate cholesterol efflux in podocytes independently of the phospholipid-transporting ATPase ABCA1, a common target for cholesterol efflux-mediated strategies, thereby reducing mitochondrial toxicity from intracellular lipid accumulation $^{69}$. None of the approaches is selective for the GFB, and the safest and most specific way to target mitochondrial health in podocytes and GECs in the setting of human disease is currently unclear. Nevertheless, a positive outcome in these ongoing clinical trials will serve as new impetus to pursue mitochondrial function improvement for GFB disease.

The rest of this section on mitochondrial dysfunction focuses on targets and approaches to intervene in two key pathways: the apolipoprotein L1 (APOL1) pathway and the coenzyme Q10 $\left(\mathrm{CoQ}_{10}\right)$ biosynthetic pathway. High-risk variants in APOL1 as well as the mutations in the $\mathrm{CoQ}_{10}$ biosynthetic pathway contribute to loss of mitochondrial function, pointing to a common pathogenic mechanism leading to GFB breakdown ${ }^{62,70,71}$.

\section{APOL1 inhibition or silencing}

The APOL1 gene region was originally identified as a risk factor in patients of recent African ancestry who were predisposed to FSGS in the setting of HIV infection. Although the genetic association was initially attributed to a neighbouring gene, $M Y H 9$, subsequent genome-wide association studies in patients with idiopathic FSGS identified the same locus but, with reasonable certainty, identified coding variants in APOL1 itself that were strongly associated with FSGS in patients of recent African ancestry ${ }^{72,73}$. These common variants in the coding region of $A P O L 1$ are known as G1 (p.S342G:I384M) and G2 (p.N388_Y389del), and are now established genetically defined risk factors for the development of kidney disease, with relative risk exceeding 20-fold when both alleles contain a variant (that is, APOL1 G1/G1, G1/G2 and G2/G2 genotypes all confer high risk). Nevertheless, many healthy individuals with the high-risk genotypes will not develop kidney disease, indicating that additional factors are required to trigger disease. Follow-up studies have associated the G1 and G2 variants with increased risk of many forms of kidney disease including hypertensive kidney disease, lupus nephritis and sickle cell disease ${ }^{74}$. The G1 and G2 variants are only found in individuals who have current or recent African ancestry, including African Caribbean, African American and Latino individuals. The prevalence of the high-risk genotype in healthy individuals of these ethnicities is $20-40 \%$. These high-risk variants are strongly enriched among patients with kidney disease in these ethnic groups ${ }^{75-77}$.

APOL1 is only found in humans, gorillas and baboons. In other primates, APOL1 protein is not produced but APOL1 can be found as a pseudogene or gene fragment, indicating evolutionary gene loss. Such an evolutionary pattern is consistent with potential toxicity from APOL1 $\left(\mathrm{REF}^{78}\right)$. Indeed, in assays performed in a range of systems including human cells and Drosophila melanogaster, the normal form of APOL1 (G0) is toxic to cells. The G1 and G2 forms have an even greater and more extensive toxic gain-of-function phenotype. 
This toxicity is partly related to the level of expression, which can be induced by inflammation or infection. Exogenous interferons and infections including HIV and COVID-19 can cause FSGS in patients with the high-risk $A P O L 1$ genotype ${ }^{79,80}$, adding further evidence that G1 and G2 are toxic in the kidney.

APOL1 is produced primarily in the liver and circulates in the high-density lipoprotein (HDL) complex, where its primary function is to kill trypanosomes that enter the bloodstream. One serious trypanosome infection, sleeping sickness, is caused by Trypanosoma brucei rhodesiense and T. brucei gambiense. Sleeping sickness arose in and is endemic to parts of central Africa. T. brucei can evade the protective effects of the normal form of APOL1. It is thought that the APOL1 G1 and G2 variants arose in Africa to specifically kill these resistant trypanosomes. APOL1 oligomerizes to form multisubunit cationic pores in trypanosome lysosomes and mitochondria, resulting in osmotic swelling and mitochondrial depolarization, which kill the organism. Lysosomal pores contribute to osmotic swelling, as does insertion of pores into the outer membrane, but pore insertion in the trypanosome mitochondrial membrane results in mitochondrial depolarization and loss of ATP production, which causes parasite death in a manner analogous to apoptosis ${ }^{81-83}$.

In addition to circulating in HDL complexes, splice variants of APOL1 that have intracellular membrane localization domains and are not released extracellularly are expressed in the vascular wall throughout the kidney, including in GECs and podocytes. This expression is induced during inflammation when interferon levels are high, particularly in response to viral infection. As with circulatory APOL1, intracellular APOL1 can be toxic to intracellular trypanosomes and may also disrupt viral packaging by inserting into membranes of intracellular vesicles $^{84}$.

Notably, the intracellular form of APOL1 is also toxic to human kidney cells ${ }^{85-87}$ and podocytes ${ }^{88,89}$. The G0 (wild type), G1 and G2 variants form oligomeric structures that can function as cation pores, producing currents that can be detected at the plasma membrane in cells expressing APOL1. However, the G1 and G2 high-risk variants selectively form oligomeric complexes in mitochondria that open the mitochondrial permeability transition pore and cause mitochondrial depolarization in human kidney cells, including podocytes. The G1 and G2 variants also traffic selectively to membranes in the endoplasmic reticulum and mitochondria in podocytes, and may directly introduce APOL1 pores into mitochondrial membranes. Therefore, although the toxic mechanism in podocytes is not fully elucidated, intracellular G1 and G2 variants selectively form multimeric complexes with pore properties in membranes that lead to loss of mitochondrial function, suggesting that mitochondria may be the primary site of toxicity from the G1 and G2 variants ${ }^{71,90}$ (FIG. 2a). Interestingly, in podocytes, the G1 and G2 variants cause loss of mitochondrial function and cell toxicity only when expressed at high levels, suggesting that a toxic threshold needs to be met $^{71,90}$.

The tendency of APOL1 to form cation pores has been reproduced in many different laboratories in cell-based and cell-free systems. Pore insertion in kidney cells is the most likely mechanism through which APOL1 injures podocytes in humans, partially because this is the innate immune mechanism through which APOL1 prevents trypanosome infections ${ }^{81}$. Nevertheless, alternative mechanisms of APOL1 variant-induced podocyte injury have been proposed, including depletion of intracellular $\mathrm{K}^{+}$and the subsequent induction of stress-activated protein kinase ${ }^{91}$, abnormal endosomal trafficking and intracellular compartment acidification ${ }^{92}$, activation of endoplasmic reticulum stress through double-stranded RNA and protein kinase R activation, which inhibits protein translation ${ }^{93,94}$, and activation of intracellular inflammasomes ${ }^{87}$. These mechanisms may be activated as a consequence of pore formation, or could contribute to podocyte injury through independent mechanisms.

The role of APOL1 in toxicity in GECs and vascular wall cells beyond the glomerulus is poorly understood. Transgenic expression of human APOL1 variants in podocytes of mice at high levels is sufficient to cause breakdown of the $\mathrm{GFB}^{87,95}$. Nevertheless, APOL1 is induced broadly in the vascular wall, not just the GFB, and APOL1 high-risk variants contribute to the development and progression of hypertensive kidney disease (nephrosclerosis), which is characterized by arteriolar vascular wall pathology as well as disease of the GFB. It is therefore possible that APOL1 contributes to vascular wall toxicity in both arterioles and peritubular capillaries of the kidney, where the pathological features of hypertensive nephrosclerosis are also prominent ${ }^{96}$.

Two therapies that inhibit the toxic functions of APOL1 are in clinical trials. Vertex Pharmaceuticals has VX-147, a small-molecule oral inhibitor of APOL1, in a phase II trial in adults with FSGS and a G1/G1, G1/G2 or G2/G2 genotype (NCT04340362). The primary end point in this trial is the percentage change in proteinuria at week 13. Ionis Pharmaceuticals and AstraZeneca have developed an ASO, delivered by systemic injection, to silence APOL1 production in the kidney, and are planning to evaluate this molecule in patients with FSGS ${ }^{95}$.

Both the small-molecule APOL1 inhibitor and the ASO targeting APOL1 are potential first in class agents for a disease with high unmet need. Major challenges for these clinical studies, however, include identifying patients who have the G1 or G2 high-risk variants, as patients with FSGS are not routinely genotyped. A second challenge for the ASO will be to demonstrate target engagement in podocytes (BOX 1). Because both of these candidate medicines block the function of APOL1, they may also block the ability of APOL1 to kill trypanosomes, although the risk of trypanosome infection is low in the developed world. Of note, an individual has been found who is homozygous null for APOL1 (REF. ${ }^{97}$ ), suggesting that blocking APOL1 will be safe in humans. This individual, who had normal kidney function, presented in India at age 45 years with an infection with Trypanosoma evansi, normally a pathogen of cattle. He was treated with suramin and made a full recovery.

APOL1 may also play a broader role in diseases of the GFB. The risk of developing other kidney diseases including hypertensive nephrosclerosis, lupus nephritis, pre-eclampsia, kidney transplant nephropathy and sickle 

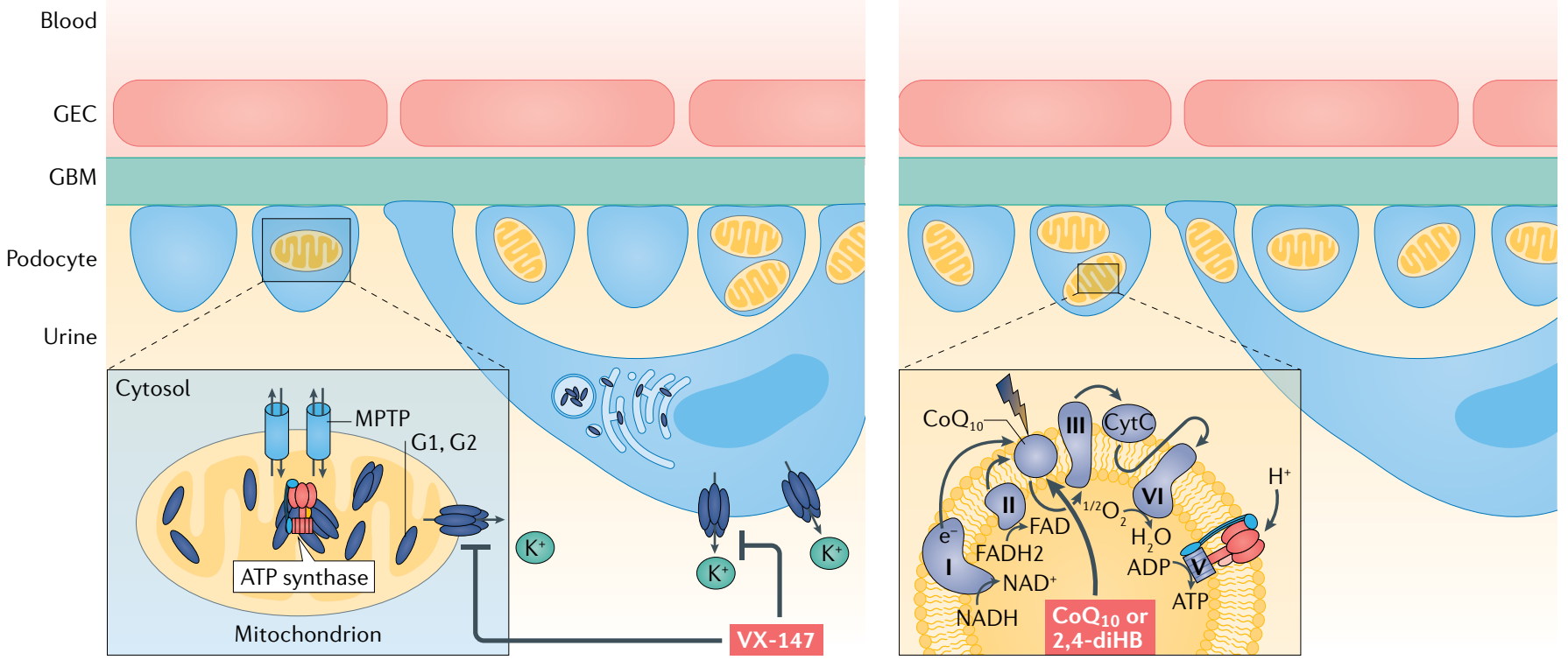

c
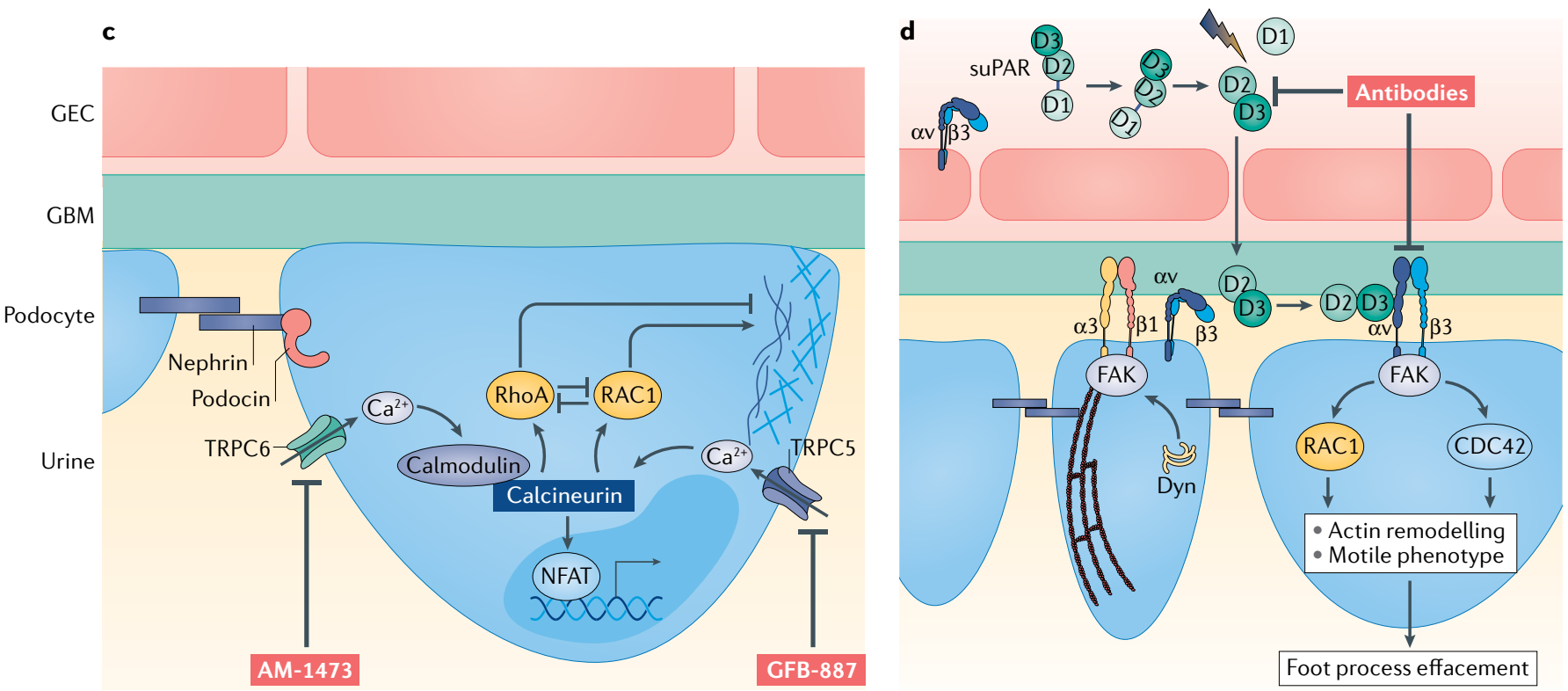

Fig. 2 | Therapeutic approaches targeting the GFB in clinical or late preclinical development. Glomerular filtration barrier (GFB), toxic mechanisms that affect podocytes and strategies to address podocyte dysfunction currently in development are shown. a | Apolipoprotein L1 (APOL1) inhibition. APOL1 genetic variants $G 1$ and $G 2$ accumulate in intracellular membranes and structures, particularly mitochondria (inset), where they form oligomers that can function as cation pores and leak cations, such as $\mathrm{K}^{+}$, across membranes. Oligomers also bind to mitochondrial inner membrane proteins, activating the mitochondrial permeability transition pore (MPTP), depolarizing mitochondria and causing mitochondrial dysfunction. VX-147 inhibits these toxic functions of APOL1. b | Coenzyme Q10 $\left(\mathrm{CoQ}_{10}\right)$ restoration. Mutations in genes encoding components of the $\mathrm{CoQ}_{10}$ biosynthetic pathway reduce levels of $\mathrm{CoQ}_{10}$, an electron acceptor of the inner membrane electron transport chain (inset), leading to mitochondrial dysfunction. Pharmacological delivery of $\mathrm{CoQ}_{10}$ or 2,4-dihydroxybenzoic acid (2,4-diHB) bypasses the defects and restores $\mathrm{CoQ}_{10}$ levels in the mitochondrial inner membrane. $\mathbf{c}$ | Short transient receptor potential channel (TRPC) inhibition. Mutant forms of TRPC6, overactivated TRPC 6 or overactivated TRPC 5 increase levels of intracellular $\mathrm{Ca}^{2+}$, which acts via calmodulin and calcineurin to activate the transcription factor nuclear factor of activated T cells (NFAT). Intracellular calcium activates RhoA (mainly via TRPC6) and RAC1 (mainly via TRPC5 and calcineurin). These signalling events remodel actin to form stress fibres and disrupt the normal actin-myosin contractile apparatus, resulting in foot process effacement, proteinuria and cell dysfunction. GFB-887 and AM-1473 inhibit TRPC5 and TRPC6, respectively. d | Soluble urokinase plasminogen activator surface receptor (suPAR) inhibition. Elevated levels of circulating suPAR, particularly a fragment containing the D2 and D3 domains, can bind to and activate $\alpha v \beta 3$ integrin at focal adhesions on podocytes. This interaction activates focal adhesion kinase (FAK), which remodels actin to form stress fibres and disrupts the normal actin-myosin contractile apparatus, resulting in foot process effacement, proteinuria and cell dysfunction. Antibodies blocking suPAR from binding to av $\beta 3$ integrin are one potential therapeutic approach when suPAR is the disease driver. CytC, cytochrome c; Dyn, dynamin; GBM, glomerular basement membrane; GEC, glomerular endothelial cell. 


\section{Box 1 Antisense oligonucleotides as candidate therapies in the GFB}

Two novel candidate therapies in clinical development to treat diseases of the glomerular filtration barrier (GFB) include antisense oligonucleotides (ASOs) against apolipoprotein L1 (APOL1) mRNA and the non-coding microRNA-21 (miR21) 68.95 . ASOs have a single-stranded DNA backbone, freely enter cells by pinocytosis and form double-stranded RNA-DNA hybrids that induce RNA cleavage by endogenous RNase $\mathrm{H}$, thereby degrading the target. Single-stranded DNA is unstable, can activate innate immune responses and is not able to pass cell membranes. Advances in DNA and RNA chemistry have resulted in substantive chemical modifications to the deoxyribose backbone that serve to stabilize the molecule, protect it from endogenous exonucleases and endonucleases, reduce its charge, render the molecules cell penetrant, reduce innate immune activation and increase affinity for target RNA, increasing activation of RNase H. Such modifications include replacing some phosphodiester bonds with phosphorothioate bonds. Other modifications increase the rigidity between bases, including 2-O-methyl, and increase affinity of the oligo for the target RNA. These modifications may contribute to translational suppression by a steric hindrance mechanism $^{224}$.

Despite these advances, a major challenge for systemically delivered ASOs is still to get the molecule to the nucleus of the target cells. Subcutaneous or intravenous delivery of ASOs results in high levels of biodistribution to the liver and kidney (mainly to hepatocytes and proximal tubule cells), with lower levels to a range of organs and tissues $^{225}$. Studies of ASO distribution to podocytes and glomerular endothelial cells (GECs) of the GFB have shown limited uptake compared with tubular structures. However, in rodent models, diseased GFBs have an increased uptake of ASOs, and studies from both lonis and Sanofi have demonstrated high degrees of silencing in the glomerulus in animal models of APOL1 disease or Alport syndrome ${ }^{68,95}$. Nevertheless, in other organ settings, such as muscle, ASO uptake and target engagement in rodent models did not translate to similar target engagement in human clinical studies. Therefore, a clear understanding of dose and glomerular cell target engagement in large animals, preferably in humans, is required before embarking on interventional studies for glomerular disease. Finally, ASOs have demonstrated mild tubular toxicity at high doses in certain settings, which has limited their advancement to clinical evaluation ${ }^{226}$. Such toxicity findings are idiosyncratic and depend on charge, backbone modifications and sequence, and therefore toxicity can be minimized through careful preclinical evaluation. A recent comprehensive study from lonis suggests that toxicity to kidney tubules is a minor risk ${ }^{227}$.

cell nephropathy is elevated in patients with APOL1 high-risk genotypes ${ }^{98,99}$. Success in proof of concept trials could lead to subsequent evaluation in these additional indications, and definition of a group of clinical presentations, all of which include GFB breakdown, as APOL1-mediated kidney diseases.

\section{Coenzyme Q10 biosynthetic pathway}

SRNS arises in childhood and is frequently identified pathologically as minimal change disease or FSGS on kidney biopsy. Whole-exome sequencing studies of children with isolated SRNS, as well as syndromes that include SRNS, have identified $>50$ genes that are thought to cause GFB breakdown; the vast majority have an autosomal recessive inheritance pattern. Several of these cluster in the proteins that regulate the biosynthesis of the lipophilic membrane constituent $\mathrm{CoQ}_{10}$, which is a benzoquinone with an isoprenoid side chain. The implicated genes include COQ2, COQ4, COQ6, COQ7, COQ9, PDSS1, PDSS2, ADCK3 and ADCK4 (REFS ${ }^{100-104}$ ). $\mathrm{CoQ}_{10}$ is found principally in the mitochondrial inner membrane, as well as in the Golgi and plasma membranes. In mitochondria, $\mathrm{CoQ}_{10}$ plays a pivotal role in mitochondrial oxidative phosphorylation, as it shuttles electrons between cyclooxygenase complexes I-II and II-III of the electron transport chain. Moreover, it has a critical antioxidant role in mitochondria because of its redox potential. The genes that are mutated in individuals with SRNS contribute to $\mathrm{CoQ}_{10}$ biosynthesis and all the mutations are associated with low levels of $\mathrm{CoQ}_{10}$, dysfunction of the electron transport chain complexes and mitochondrial dysfunction.

To understand the importance of $\mathrm{CoQ}_{10}$ in GFB disease, several studies have inserted human mutations into mice or specifically disrupted individual COQ genes somatically in mouse podocytes. Loss of function of these $\mathrm{CoQ}_{10}$ biosynthetic genes in podocytes is sufficient to recapitulate the hallmarks of human disease: nephrotic syndrome and FSGS ${ }^{57,105}$. Importantly, in these studies, podocytes with COQ gene mutations have abnormal mitochondrial morphology, dysfunction of the electron transport chain and loss of inner mitochondrial membrane potential. Podocyte cytoskeletal proteins are also reduced, in keeping with a role for mitochondria in maintaining foot processes (FIG. 2b).

Several clinical case series have now tested a therapeutic role for oral $\mathrm{CoQ}_{10}$ or its synthetic analogue idebenone, and demonstrated that SRNS and FSGS can be stabilized or reduced in children if the disease is treated fairly early in its course ${ }^{106,107}$ (FIG. 2b). $\mathrm{CoQ}_{10}$ and idebenone have been used at high doses $\left(30 \mathrm{mg}^{-1} \mathrm{~kg}^{-1} \mathrm{day}^{-1}\right)$ and have limited oral bioavailability owing to the lipophilic nature of $\mathrm{CoQ}_{10}$, which limits its transport to the mitochondrial inner membrane. The study of the $\mathrm{CoQ}_{10}$ biosynthetic pathway has identified an intermediate, 2,4-dihydroxybenzoic acid (2,4-diHB), that could be more therapeutically useful than $\mathrm{CoQ}_{10}$. Hydrophilic properties give 2,4-diHB superior bioavailability to exogenously supplied $\mathrm{CoQ}_{10}$, and exogenous 2,4-diHB can also bypass many of the loss-of-function mutations and normalize $\mathrm{CoQ}_{10}$ levels ${ }^{108,109} \cdot 2,4$-diHB is found naturally in certain foods including coffee and avocados, and has a good safety profile. If given in drinking water to mice with COQ6 or ADCK4 mutations, 2,4-diHB reversed the progression of disease; the compound also normalized mitochondrial function in cells from a patient with a homozygous COQ7 mutation ${ }^{57,109,110}$. This substrate will likely be tested more comprehensively in human studies in the near future, offering the possibility of halting or reversing disease in children with mutations in COQ genes. Additional substrates, including vanillic acid, may also bypass the $\mathrm{CoQ}_{10}$ biosynthetic pathway and elevate levels of this key membrane quinone. One recent study of $\mathrm{CoQ}_{10}$ deficiency in mice identified that the podocytes of these mice had elevated levels of polyunsaturated fatty acids, which can activate stress-activated kinase pathways. Treatment with GDC0879, a small-molecule inhibitor of the BRAF kinase pathway, rescued podocyte injury and kidney function in those models. These observations suggest that mitochondrial dysfunction in podocytes, as a result of $\mathrm{CoQ}_{10}$ deficiency, may confer toxicity through excessive production of fatty acid metabolites as well as dysfunction of the electron transport chain ${ }^{111}$.

\section{Outlook on mitochondrial therapies}

The two promising areas of therapeutic intervention in patients highlighted here support an emerging picture that GFB breakdown can result directly from 
mitochondrial dysfunction. The selective role of these genetically defined targets in kidney disease suggests that, similar to neurons and myofibres, podocyte function is highly dependent on correctly functioning mitochondria. The strong validation of APOL1 as a target for kidney disease and the high prevalence of $A P O L 1$ variants in patients with kidney disease suggest that APOL1 inhibition has the potential to have broad effects on kidney health in humans. Recent studies point to additional mitochondrial functions in podocytes and GECs, such as the control of fatty acid metabolism ${ }^{111}$, which could uncover new mitochondrial targets. Novel biomarkers are required to study target engagement to guide drug dosing in clinical trials.

\section{Targeting actin and myosin}

The majority of pathological mutations in genes identified to cause isolated FSGS or SRNS are reported to regulate the assembly, turnover and degradation of the actin cytoskeleton or regulate its attachment at the cell surface ${ }^{46,48}$. The formation and maintenance of podocyte tertiary processes requires complex actin filament assembly and disassembly, and integration at focal adhesions and slit diaphragm protein complexes. Expression of mutated forms of genes including ACTN4, INF2, AHRGAP24 and AHRGDIA in animal models directly interferes with podocyte tertiary process formation ${ }^{112-114}$. For example, the AHRGDIA gene product is a dissociation inhibitor of the GTPase family (Rho-GDIa), which locks RhoA and another Rho family member, RAC1, in their inactive states. RhoA functions dynamically in the assembly of actin filaments by activating a group of actin polymerizing proteins called formins. Mutant forms of Rho-GDIa fail to interact with RhoA; podocytes expressing these proteins adopt a hypermigratory phenotype and can no longer maintain the GFB. Another protein that has been found to be mutated, inverted formin 2 (INF2), is a member of the actin polymerizing family of formin proteins and plays important roles in cell spreading. Mutations in INF2 act dominantly and fail to promote normal cell spreading ${ }^{115}$.

Mutations in numerous proteins involved in actinmyosin dynamics are found in individuals with FSGS or SRNS, and these dynamics are vital to maintaining the GFB. Collectively, these findings suggest that normalizing dysfunctional actin-myosin dynamics should have therapeutic utility. Drugging these proteins directly, however, is challenging because of the ubiquitous nature of this bioactive cytoskeleton. Nevertheless, the presence of isolated GFB disease caused by loss-of-function mutations in individual genes of the actin-myosin machinery suggests that facets of its function that are specific to podocytes and GECs could be identified. To that end, studies of podocyte-specific actin-myosin machinery components have identified that a complex containing actin-related protein 2 (ARP2) and ARP3 is central to tertiary process formation through the formation of branched actin networks. In particular, ARP3 plays an important role in maintaining normal podocyte architecture. In the absence of ARP3, the actin-myosin machinery is overactivated, leading to stress fibres and, ultimately, to cell detachment ${ }^{116}$. It is currently unclear whether ARP2 or ARP3 function could be therapeutically restored in disease settings.

Another potentially druggable target is dynamin. Dynamin, encoded by DNM1, DNM2 and DNM3, is another GTPase that has well-recognized roles in clathrin-mediated endocytosis. This process forms multimeric ring structures that pinch off sections of cell membranes to form endosomes ${ }^{117}$. Dynamin has also been reported to directly interact with filamentous actin and promote actin assembly, particularly in the formation of filopodia, membrane ruffles and cell spreading ${ }^{118}$. The dynamin-mediated actin assembly seems to be independent of Rho-GTPases and formins. Several studies suggest that dynamin oligomerization caused either by a gain-of-function point mutation in DNM1 or by the use of a divalent small molecule, Bis-T-23, that stimulates actin oligomerization can overcome both genetic and environmental defects in actin assembly in podocytes, restore foot process formation and restore the $\mathrm{GFB}^{119}$. Bis-T-23 was originally identified as an inhibitor of dynamin-mediated endocytosis and has poor drug-like properties $^{120}$, and therefore its precise mode of action in restoring the GFB is unclear. Moreover, mutations in dynamin genes have not been associated with diseases of the GFB in humans. Nevertheless, if small molecules that selectively promote dynamin oligomerization can be developed and overcome potential safety liabilities, they may have broad utility in restoring GFB function.

Therapies targeting other aspects of the actin-myosin machinery are further along in development. In the following sections, we discuss therapies in development that target channels and receptors that directly regulate the function of the actin-myosin cytoskeleton in the GFB. In particular, molecules targeting short transient receptor potential channels (TRPCs), roundabout homologue (ROBO) proteins, integrins and endothelin receptors to treat GFB disease are currently in clinical or late preclinical evaluation. The molecules presented are a selection of druggable targets for therapeutic intervention with varying degrees of human target validation.

\section{Podocyte-directed therapies}

TRPC6 and TRPC5. Approximately 1-2\% of patients with FSGS have mutations in TRPC6, which has an autosomal dominant inheritance pattern. Similar to other TRPCs, TRPC6 is a non-selective cation channel that permits $\mathrm{Ca}^{2+}$ entry into the cell upon activation by stretch, oxidative stress or local factors including angiotensin release. Influx of $\mathrm{Ca}^{2+}$ into podocytes acts as a second messenger in microdomains of the cell, as it regulates actin-myosin assembly and activates transcription factors such as nuclear factor of activated $\mathrm{T}$ cells $(\text { NFAT })^{121}$. TRPC6 localizes to foot processes, and its expression is increased in a range of kidney diseases. Although many patients have mutations that increase channel activity, others have mutations that do not increase channel activity ${ }^{122}$. Nevertheless, a common feature of disease-associated mutations in TRPC6 is that they increase activation of calcineurin, a calciumdependent phosphatase that regulates the activity of ERK1/2 and NFAT family member NFATc1 (REF. ${ }^{123}$ ). Mutant forms of TRPC6 activate NFATc1 through a 
dominant mechanism ${ }^{124,125}$ that rapidly leads to podocyte effacement and proteinuria ${ }^{126}$. TRPC6 mutations also cause defects in the actin cytoskeleton by acting through the Rho-GTPases, Rho and RAC, and hence contribute to abnormalities of the actin-myosin machinery that maintains foot processes and adhesion to the basement membrane (FIG. 2c). TRPC6 mutations in podocytes contribute to cell detachment and cell death during cell stress ${ }^{127}$. TRPC6 transgenic mice expressing extra copies of wild-type or mutant forms of TRPC6 develop albuminuria $^{128}$. Although the number of patients who have TRPC6 mutations is small, there are several studies in animal models suggesting that wild-type TRPC6 is an important intermediate in the kidney disease cascade downstream of angiotensin receptor type 1 activation, raising the possibility that TRPC6 inhibition may be useful in kidney diseases with high angiotensin levels, such as hypertensive kidney disease ${ }^{129}$.

Amgen have generated highly potent and selective inhibitors of TRPC6 channel activity, including AM-1473. These inhibitors used novel cryogenic electron microscopy structures of the channel and patient-derived pathological mutations to identify a novel binding site that can be used to selectively block TRPC6 function ${ }^{130}$ (FIG. 2c). As yet, no clinical studies in patients have been announced. Selecting the optimal patient population to evaluate such inhibitors may be challenging; genotyping studies of cohorts of patients with FSGS indicate that TRPC6 mutations are rare. An alternative patient population in which TRPC6 inhibition may be assessed is those with hypertensive kidney disease, which has high circulating angiotensin levels. In preclinical studies, overactivity of wild-type TRPC6 has been reported to drive disease pathology $y^{129}$. TRPC6 activity appears to require calcineurin and NFAT, a pathway inhibited by the calcineurin inhibitors cyclosporin and tacrolimus. As calcineurin inhibitors are already in use for FSGS, novel TRPC6 inhibitors may not be additive with existing therapies, and will therefore be required to show superior characteristics or a cleaner safety profile. It should be noted that TRPC6 channels play a role in vascular smooth muscle tone and cardiac contractility in pathological settings, so it will be interesting to understand whether TRPC6 inhibition has negative inotropic or hypotensive effects ${ }^{124}$.

A second TRPC family receptor, TRPC5, has also been reported to play a role in calcium and calcineurin signalling in podocytes ${ }^{131}$. TRPC6 has been reported to form a molecular complex with RhoA and inhibit cell migration, whereas TRPC5 has been reported to form a molecular complex with RAC1 and promote cell migration ${ }^{112,113,131}$. Unlike TRPC6, no human TRPC5 mutations have been reported to be associated with kidney disease, and levels of expression of TRPC5 do not seem to be regulated in response to disease ${ }^{132}$. Nevertheless, mutations in genes encoding regulatory proteins result in RAC1 overactivation and cause human disease $^{112,113}$, and TRPC5 loss-of-function in mice protects podocytes and the GFB in settings where podocytes are injured ${ }^{133}$. Selective small-molecule inhibitors of TRPC5 calcium flux have been developed, and demonstrated efficacy in a transgenic rat model of FSGS as well as in angiotensin-driven hypertensive kidney disease in rats $^{134}$. Administration of a small-molecule TRPC5 inhibitor during established GFB breakdown reduced the rate of progression of kidney disease and preserved GFB integrity ${ }^{134}$.

Goldfinch Bio has developed a small molecule, GFB-887, that selectively inhibits TRPC5. The company has initiated a phase II basket study trial (NCT04387448) to evaluate this compound in patients with SRNS, FSGS or diabetic nephropathy (FIG. 2c). The primary end point is the percentage change in the ratio of urine protein to creatinine at 12 weeks. The questions concerning patient selection and demonstration of superiority over calcineurin inhibitors posed above for TRPC6 inhibition also apply to studies of TRPC5 inhibition. It also remains unclear whether TRPC5 inhibition alone will be effective if TRPC6 currents and calcineurin activation remain intact in podocytes. Although TRPC5-RAC1 and TRPC6-RhoA reportedly have opposite effects on podocyte migration ${ }^{131}$, forced activation of either RhoA or RAC1 in podocytes can cause GFB breakdown. It therefore remains unclear whether TRPC5 inhibition will be different from TRPC6 inhibition in the diseased GFB of patients ${ }^{131,135}$. One potential solution Goldfinch Bio has undertaken is to identify patients with elevated RAC1 signalling as an exploratory biomarker for evaluation of patients receiving GFB-887. A urinary biomarker may identify patients who will be responsive and guide further evaluation of the study drug.

Soluble urokinase plasminogen activator surface receptor inhibition. Urokinase plasminogen activator surface receptor (uPAR) is a glycosylphosphatidylinositolanchored receptor that regulates plasminogen activation. Activated plasminogen is converted into plasmin and acts in clot lysis. However, uPAR is multifaceted as it can be cleaved to soluble forms, known as suPAR. suPAR isoforms have been identified as candidate circulating factors that cause disruption of the GFB in patients whose severe proteinuria recurs after kidney transplantation ${ }^{136}$ (FIG. 2d). Recurrent FSGS (rFSGS) is rare but can rapidly lead to kidney failure of newly transplanted kidneys in patients who previously had FSGS. This recurrence can be explained by a circulating factor, produced by the transplant recipient, which is toxic to the kidney and likely causes or contributes to the primary disease $\mathrm{e}^{136}$. suPAR is one candidate for this toxic factor, as it was identified from the plasma of patients with rFSGS. Whereas full-length suPAR has little toxicity, some isoforms are susceptible to protein cleavage, which can generate toxic fragments such as D2-D3 $\left(\right.$ REF. $\left.^{137}\right)$. The binding of suPAR fragments to $\alpha v \beta 3$ integrin activates downstream signalling events at focal adhesions of podocytes. In gain-of-function and loss-of-function model systems in mice, fragments of suPAR confer glomerular toxicity, albeit over weeks and months ${ }^{137}$. The toxicity requires a second hit, in the form of hyperlipidaemia or another glomerular toxin, for disease to manifest ${ }^{137}$.

The integrin $\alpha v \beta 3$ has long been implicated in cell motility and vascular permeability, but has been most widely studied in thrombosis, arthritis, osteoporosis, inflammation, angiogenesis and viral entry. Activation of 
a

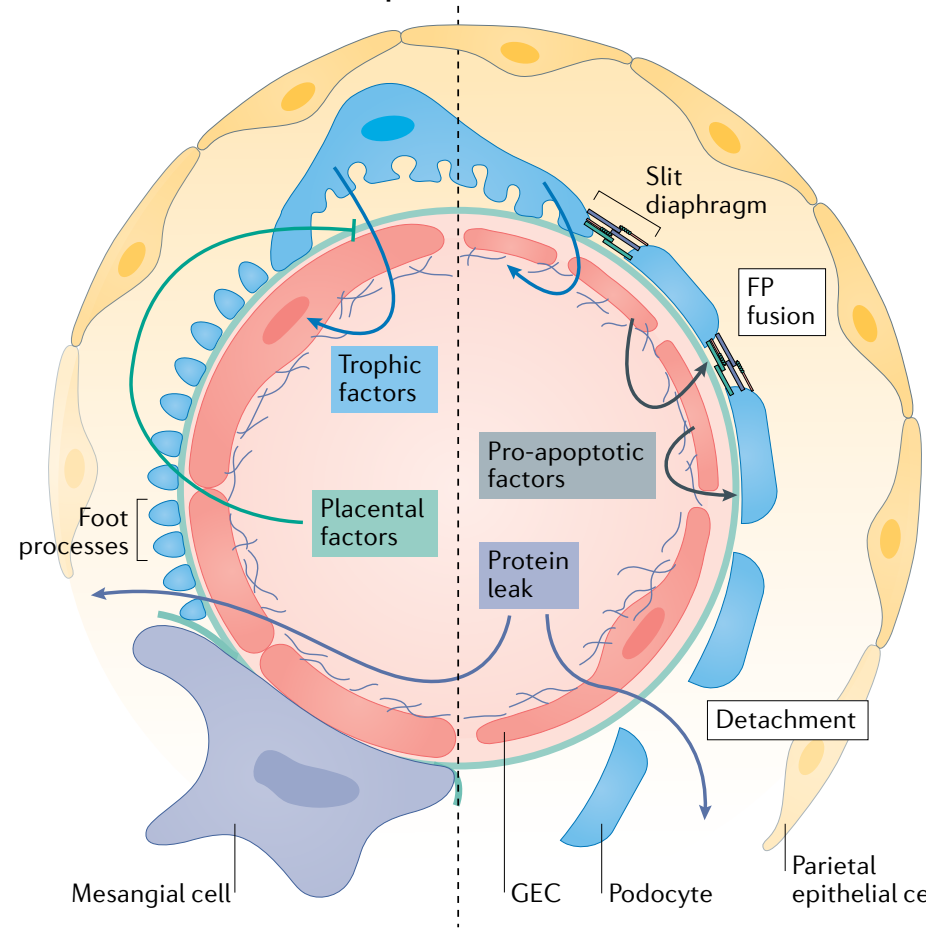

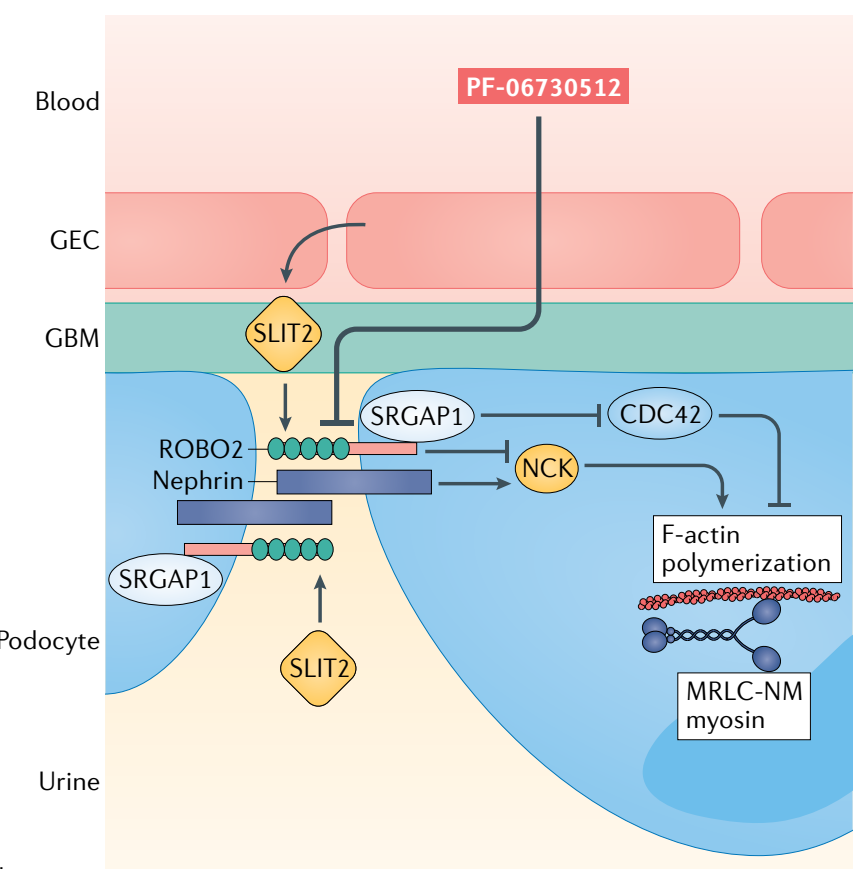

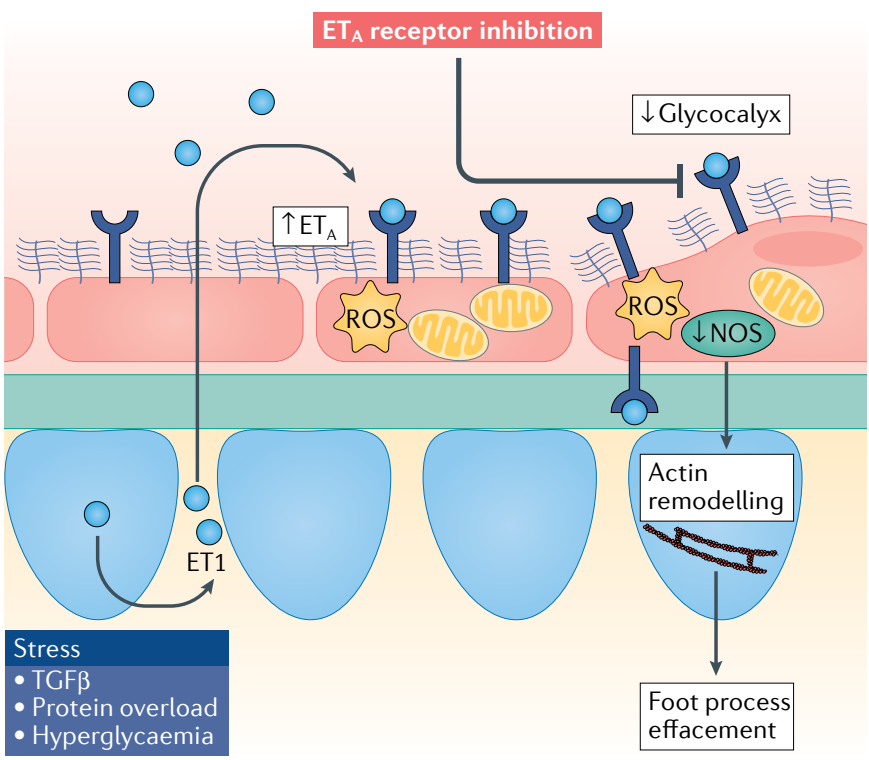

$\alpha v \beta 3$ by one of its many ligands, including fibronectin or fibrinogen, changes the cellular cytoskeleton by activating focal adhesions. Focal adhesions are macromolecular complexes through which force can be transmitted from the extracellular matrix to the cell. Such activation may also depend on local shear stress, such as that created by flow ${ }^{138}$. $\alpha v \beta 3$ is widely expressed by various cell types in the body. It is detected at low levels on podocytes as well as other vascular cells, including endothelium. As well as ligands such as fibronectin and fibrinogen, av $\beta 3$ may become activated by binding suPAR.

The plasma suPAR concentration has been identified as a robust biomarker of FSGS and other kidney diseases ${ }^{139,140}$. In addition, its levels predict the development and progression of kidney and cardiovascular diseases in very large patient populations ${ }^{141}$, highlighting the utility of suPAR as a biomarker that predicts outcomes in a number of diseases. suPAR levels, however, do not predict which patients will develop rFSGS after transplantation, and neither do they identify paediatric patient populations with FSGS or SRNS ${ }^{142,143}$. Blood levels of suPAR do not correlate well with the severity of the phenotype, suggesting that suPAR requires additional factors to play a toxic role. Consistent with this hypothesis, several investigators have not reproduced the toxic gain of function that has been reported in 
$\varangle$ Fig. 3 | Therapeutic approaches targeting podocyte-endothelial cell crosstalk in clinical or late preclinical development. a |Glomerular capillary loops showing injury to glomerular endothelial cells (GECs) from toxins, cellular stress or depletion of podocyte-derived trophic factors. Such injury can result in endotheliosis (left), as observed in pre-eclampsia. Endothelial injury may also result in the release of GEC-derived pro-apoptotic factors (right) causing focal segmental glomerulosclerosis (FSGS). In both settings there is breakdown of the glomerular filtration barrier (GFB). Foot process (FP) fusion reduces the number of slit diaphragms, causing protein leakage and, ultimately, podocyte detachment. Strategies to rebalance podocyte trophic factors or inhibit endothelial cell-derived apoptotic factors are under evaluation. Adapted from $\mathrm{REF}^{223} \cdot \mathbf{b} \mid$ Roundabout homologue 2 (ROBO2) inhibition. Endothelial cell-derived SLIT2 activates $\mathrm{ROBO} 2$ in podocytes to negatively regulate actin polymerization and nonmuscle myosin activity, acting as a counterbalance to nephrin signalling. $\mathrm{ROBO} 2$ signals via the adaptor protein slit-robo rho GTPase activating protein 1 (SRGAP1) and protein intermediates $\mathrm{NCK}$ and $\mathrm{CDC} 42$. A ROBO2-crystallizable fragment $(\mathrm{Fc})$ fusion protein (PF-06730512) blocks ROBO2 activation, enhancing nephrin function in disease states. c| Endothelin type $\mathrm{A}\left(\mathrm{ET}_{\mathrm{A}}\right)$ receptor inhibition. In response to podocyte stress or injury, $\mathrm{ET}_{\mathrm{A}}$ receptor is upregulated on GECs and the ligand endothelin (ET1) is released from podocytes. $\mathrm{ET}_{\mathrm{A}}$ receptor activation in adjacent $\mathrm{GEC}$ s causes mitochondrial depolarization, resulting in damage from reactive oxygen species (ROS) and a reduction in nitric oxide synthase (NOS). These changes cause loss of GEC fenestrae and degradation of the glycocalyx. Such GECs release pro-apoptotic factors, which in turn cause podocyte stress fibre formation and, ultimately, cell depletion. $\mathbf{d}$ | Soluble fms-like tyrosine kinase 1 (sFLT1) inhibition. In pre-eclampsia, high levels of sFLT1, an endogenous inhibitor of vascular endothelial growth factor (VEGF) signalling, are released from the placenta into the circulation. Differentiation and maintenance of specialized functions of GECs relies on high levels of VEGF, released from podocytes, engaging VEGF receptor 2 (VEGFR2) on GECs. High concentrations of sFLT1 'mop up' podocyte VEGF and block this tonic signal, causing GEC swelling and dysfunction. Although podocyte foot processes remain intact, the glycocalyx and fenestrae of GECs are disrupted, which is sufficient to compromise the GFB. A silencing small interfering RNA (siRNA) directed at the placenta reduces circulating levels of sFLT1 in the setting of pre-eclampsia. GBM, glomerular basement membrane; MRLC-NM myosin, non-muscle myosin regulatory light chain.

animal models ${ }^{144}$. Overall, through binding to and activating av $\beta 3$, suPAR is likely an important factor in GFB breakdown in some patients.

Several approaches to targeting suPAR are being considered (FIG. 2d). Walden Biosciences is developing neutralizing antibodies that prevent suPAR fragments from interacting with $\alpha v \beta 3$ integrin but has not yet announced clinical studies ${ }^{145}$. The molecular hypothesis predicts that inhibition of $\alpha v \beta 3$ integrin with antibodies or small-molecule inhibitors could also offer therapeutic benefit. Of note, Janssen/Vascular Therapeutics' VPI-2690B is an antibody that blocks av $\beta 3$ signalling and has been evaluated for protection of the GFB in diabetic nephropathy. The study completed in 2017 but the results have not been disclosed. Several other small-molecule, peptide and antibody inhibitors of $\alpha v$ integrins (which may include $\alpha v \beta 3, \alpha v \beta 5$ or $\alpha v \beta 1$ ), including MK-0429, cilengitide (EMD121974) and Vitaxin (LM609), have been generated and evaluated for a number of indications, particularly vascular growth in cancers. All have failed to show significant efficacy and are no longer being developed. Although none of them has been tested in rFSGS, these completed studies indicate that $\alpha v \beta 3$ integrin inhibition is unlikely to be successful. It will be important to understand whether suPAR has differential binding and activation of $\alpha v \beta 3$ integrin or whether other receptors are responsible for the pathological effects of suPAR on the GFB.

The challenges for demonstrating efficacy in humans are significant. Which patients with FSGS have
suPAR-driven disease? Should the many patients with FSGS with underlying mutations that cause podocyte dysfunction be excluded? Despite the controversies surrounding the precise toxic role of suPAR, therapies targeting this cytokine merit safe evaluation in patients.

\section{Targeting podocyte-GEC crosstalk}

As in many other systems, interdependence exists between cells in the GFB, which cooperate to maintain the structure and function of the barrier. Research has begun to uncover the broad reach of intercellular communications between podocytes and GECs. For example, podocytes and GECs secrete factors and extracellular vesicles that deliver detrimental signals or block trophic signals, and thereby disrupt the barrier and promote disease (FIG. 3a). Intercellular signalling molecules that mediate survival or dysfunction of podocytes and GECs have also emerged as promising therapeutic targets for GFB diseases. Some of these molecules have progressed to clinical evaluation, underscoring the importance of intercellular signalling to health and pathology.

ROBO2-SLIT2 inhibition. ROBOs are receptors found on cell surfaces. ROBOs and their protein ligands, SLITs, were first described as repulsive factors in axon guidance. However, ROBOs and SLITs are more widely expressed and regulate repulsion and guidance elsewhere, including in the vasculature. Loss-of-function mutations in $\mathrm{ROBO} 2$ and its adaptor protein, slit-robo rho GTPase activating protein 1 (SRGAP1), are associated with congenital abnormalities of kidney and urinary tract syndrome, which is characterized by vesicoureteral reflux ${ }^{146,147}$. SLIT2 binds to ROBO2 to direct repulsive signalling through SRGAP1 and limit branching of the ureteric bud, and $\mathrm{ROBO} 2$ deficiency results in ectopic or supernumerary collecting ducts ${ }^{148}$. In the glomerulus, SLIT2 and SLIT3 are secreted proteins predominantly derived from GECs and mesangial cells, whereas ROBO2 is exclusively expressed by podocytes. Therefore, GECs and mesangial cells play an active role in regulating tertiary processes and slit diaphragm maintenance through cellular crosstalk ${ }^{149,150}$ (FIG. 3b).

ROBO2 and SRGAP1 are also highly expressed in developing podocytes and expressed at foot processes of mature podocytes, where $\mathrm{ROBO} 2$ signalling inhibits nephrin-mediated polymerization of actin, which is required to form tertiary processes ${ }^{149}$. $\mathrm{ROBO} 2$, through interaction with SRGAP1, downregulates the GTPase activity of a Rho GTPase, CDC42, which in turn counteracts actin polymerization, thereby directly inhibiting foot process extension and function. Loss-of-function of $\mathrm{ROBO} 2$ in mouse podocytes results in wider tertiary processes, but the GFB is preserved until adulthood, whereupon very mild dysfunction of the GFB can be detected $^{149}$. However, when ROBO2 was mutated in mice with concurrent nephrin mutations, mice were protected from proteinuria and disease ${ }^{149}$ (FIG. 3b). Rare mutations in the slit diaphragm protein nephrin cause severe childhood-onset SRNS and FSGS. Nephrin has a structural role in the slit diaphragm and also signals via $\mathrm{NCK}$ and CDC42 to promote actin polymerization to maintain foot processes. In human diseases of the GFB, 
nephrin levels and function are consistently diminished. Inhibition of ROBO2 in such settings would be predicted to counteract disruption of the GFB as a result of diminished nephrin function.

ROBO2 and SRGAP1 directly inhibit foot process elongation by a second mechanism. $\mathrm{ROBO} 2$ and SRGAP1 form a complex with non-muscle myosin II proteins, which comprise two heavy chains and two light chains. There are three isoforms of heavy chains encoded by three genes, MYH9, MYH10 and MYH14 $\left(R E F{ }^{150}\right)$. The non-muscle myosins work with actin to maintain podocyte foot processes, the slit diaphragm and the GFB. Patients with autosomal dominant mutations in $M Y H 9$ can present with glomerular disease, including haematuria, proteinuria, nephrotic syndrome and foot process effacement associated with macrothrombocytopenia and hearing loss ${ }^{151}$. There is variable penetration of the phenotype, which can occur in childhood but often presents in adults. The disease is thought to be caused by loss-of-function and haploinsufficiency of MYH9. MYH9 ${ }^{-1-}$ mice do not develop kidney disease but are more susceptible to injury-induced kidney disease ${ }^{152}$. In the setting of salt loading, MYH9-deficient mice develop proteinuria; concurrent $\mathrm{ROBO} 2$ deficiency protects mice from proteinuria. Absence of $\mathrm{ROBO} 2$ in podocytes in mice results in partial protection from a range of toxic and environmental injuries to the GFB including protamine sulfate and nephrotoxic serum ${ }^{153}$. These findings point to regulation of podocyte actin-myosin contractile apparatus playing a significant role in regulating the GFB in health and disease.

Pfizer has developed a protein that is a fusion between ROBO2 and the crystallizable fragment $(\mathrm{Fc})$ antibody domain. This protein, PF-06730512, blocks the interaction of ROBO2 with SLIT2 when administered intravenously. Pfizer is currently evaluating the activity of PF-06730512 in phase II clinical studies in adults with FSGS (NCT03448692) (FIG. 3b). The primary end point is the percentage change in the ratio of urinary protein to creatinine at week 13. Fc fusion proteins have been used successfully to block receptor signalling in other human diseases, but generally have to be delivered intravenously.

Challenges to the $\mathrm{ROBO} 2-\mathrm{Fc}$ fusion clinical trial include the risk of redundancy, as both ROBO1 and ROBO4 are also expressed in the kidney, and the need to understand which patients have $\mathrm{ROBO} 2$-dependent protein leak, as the level of expression of $\mathrm{ROBO} 2$ may be an important factor in its pathogenic role. In addition, because $\mathrm{ROBO} 2$ deficiency in mouse podocytes itself results in adult-onset mild proteinuria, it will be important to understand the therapeutic window, dose-response curve and pharmacodynamics of the fusion protein in humans to avoid excessively blocking the pathway.

Endothelin receptor A inhibition. Endothelin 1 (ET1; also known as Edn1) is a potent vasoconstrictor that acts by binding to endothelin type $\mathrm{A}\left(\mathrm{ET}_{\mathrm{A}}\right)$ receptor and $\mathrm{ET}_{\mathrm{B}}$ receptor, which are expressed in a wide variety of cell types ${ }^{154}$. Increased urinary ET1 excretion has been observed in patients with diabetic kidney disease or
FSGS $^{155-157}$. Variation in the regulation or expression of $\mathrm{ET} 1$ or $\mathrm{ET}_{\mathrm{A}}$ receptor in humans is associated with vascular diseases including coronary artery disease, hypertension, renal artery stenosis, arterial dissection and migraine, but no genetic association with disease of the GFB has been demonstrated ${ }^{158,159}$.

ET1 has been investigated by infusion in healthy humans ${ }^{160}$ and rodents ${ }^{161}$ in the setting of diabetes ${ }^{162}$ and pre-eclampsia ${ }^{163}$, and in rodent models of FSGS ${ }^{157}$. Whereas ET1 activation of $\mathrm{ET}_{\mathrm{B}}$ receptor results in vasodilation, the $\mathrm{ET} 1-\mathrm{ET}_{\mathrm{A}}$ receptor system results in vasoconstriction and systemic blood pressure. Infusion of ET1 directly increased glomerular permeability to albumin and renal inflammation via $\mathrm{ET}_{\mathrm{A}}$ receptor activation, independent of changes in arterial pressure ${ }^{164}$.

ET1-induced contraction of vessels occurs through RhoA activity and phosphatidylinositol 3-kinase $(\mathrm{PI} 3 \mathrm{~K})$ activity, which is induced downstream of $\mathrm{ET}_{\mathrm{A}}$ receptor and increases the level of myosin light chain phosphorylation ${ }^{165}$. Activation of RAC1 by ET1 contributes to the hypertrophic response of cardiac myocytes by modulating mitogen-activated protein kinase (MAPK) signalling cascades ${ }^{166}$. ET1 can also mediate disruption of the F-actin cytoskeleton and promote dysfunction of the slit diaphragm through activation of the Rho and $\mathrm{PI} 3 \mathrm{~K}$ pathways via $\mathrm{ET}_{\mathrm{A}}$ receptor in a mouse immortalized podocyte line ${ }^{167,168}$. These preclinical studies point to an important role for ET1 in proteinuric chronic kidney diseases and highlight the potential for $\mathrm{ET}_{\mathrm{A}}$ receptor inhibition in maintenance of the $\mathrm{GFB}^{169,170}$.

In the glomerulus, ET1 and its receptors are expressed by mesangial cells, GECs and podocytes and have a specific local role in maintaining the GFB. ET1 has been reported to signal via both $\mathrm{ET}_{\mathrm{A}}$ and $\mathrm{ET}_{\mathrm{B}}$ receptors in podocytes and drive the development of glomerulosclerosis ${ }^{171}$. In hypertensive rats, selective $\mathrm{ET}_{\mathrm{A}}$ receptor blockade prevented renal fibrosis and glomerulosclerosis via inhibition of RhoA and Rho-associated protein kinase (ROCK) activity ${ }^{172,173}$. An important role for $\mathrm{ET} 1-\mathrm{ET}_{\mathrm{A}}$ receptor signalling in the GFB was highlighted by three findings: $\mathrm{ET}_{\mathrm{A}}$ receptor expression was only detected in GECs of human biopsies and mouse kidneys in samples that had signs of diabetic kidney disease ${ }^{62}$ or $\mathrm{FSGS}^{61,174} ; \mathrm{ET}_{\mathrm{A}}$ receptor-expressing GECs exhibit mitochondrial oxidative damage; and pre-proendothelin (ET1 precursor protein) is produced locally in high quantities by adjacent podocytes ${ }^{61,64}$. In these studies, ET1 released from podocytes induced $\mathrm{ET}_{\mathrm{A}}$ receptor-dependent signalling in GECs. This signalling caused endothelial dysfunction characterized initially by loss of GEC fenestrations and loss of the glycocalyx, followed by podocyte injury and loss of the $\mathrm{GFB}^{61,62,64}$ (FIG. 3c). Selective $\mathrm{ET}_{\mathrm{A}}$ receptor antagonism prevented loss of the glycocalyx in mice with type 2 diabetic kidney disease and ameliorated proteinuria ${ }^{175,176}$. The studies found an unexpected interdependence between GECs and podocytes of the GFB in which $\mathrm{ET} 1-\mathrm{ET}_{\mathrm{A}}$ receptor directly contributes to loss of the GFB, and support the notion that selective pharmacological inhibition of $\mathrm{ET}_{\mathrm{A}}$ receptor can directly preserve the GFB.

Endothelin receptor antagonists have been evaluated in patients with proteinuric glomerular diseases 
including diabetic kidney disease and FSGS, have demonstrated encouraging anti-proteinuric effects and may stabilize kidney function ${ }^{177,178}$. The SONAR phase III clinical trial, which tested the selective $\mathrm{ET}_{\mathrm{A}}$ receptor antagonist atrasentan versus placebo in people with type 2 diabetic kidney disease, showed a 35\% reduction in the relative risk of composite renal events ${ }^{179}$. The trial was prematurely terminated owing to fluid retention-related adverse events; however, adverse events related to fluid retention may be less of an issue in patients with little or no cardiovascular disease. Atrasentan is entering new phase III studies in rare diseases of the GFB (NCT04573920). In FSGS, the phase II DUET trial found that sparsentan, a combined $\mathrm{ET}_{\mathrm{A}}$ receptor inhibitor and angiotensin receptor blocker (ARB), reduced proteinuria (urinary protein to creatinine ratio) by $\sim 50 \%$ compared with ARB treatment alone in people with primary FSGS ${ }^{180}$. In the 84-week open-label extension, the investigational agent was well tolerated, stabilized kidney function and reduced proteinuria to near normal levels in $40 \%$ of those studied. Following the success of the proof of concept study, a phase III DUPLEX trial has recruited 300 patients with FSGS with a primary end point of change in the estimated glomerular filtration slope, which measures the change in glomerular function over time (NCT03541174). An interim analysis of the proteinuria effect will be available in 2021. ET $_{A}$ receptor inhibitors are being pursued as a treatment for other glomerular and renal vascular diseases, including IgA nephropathy (phase III PROTECT; NCT03762850) and in patients with kidney disease caused by systemic sclerosis (phase II ZEBRA; NCT02047708).

\section{Outlook on actin-myosin therapies}

The expanding list of mutations in genes involved in actin-myosin assembly and disassembly provides important insight into the underlying functions of podocytes in the GFB. Together with evidence from drug toxicities that cause kidney disease by challenging the actin cytoskeleton architecture in podocytes ${ }^{181}$, or from other acquired perturbations in cytoskeleton regulation in podocytes, targeted therapies that stabilize actin-associated proteins in podocytes hold the potential to maintain the structural integrity of podocytes and treat GFB diseases.

\section{Targeting systemic factors: sFLT1}

Disruption to the GFB can be precipitated by systemic physiological changes. For example, pre-eclampsia, a serious condition that occurs in $2-8 \%$ of all pregnancies, is responsible for thousands of deaths annually, particularly in low and middle-income countries, where an infrastructure of surveillance and management is not readily available. Proteinuria is one of the diagnostic criteria of pre-eclampsia; others include hypertension and oedema. Approximately $25 \%$ of patients with pre-eclampsia have high-grade proteinuria and nephrotic syndrome. Biopsies of pre-eclamptic kidneys typically show endotheliosis and thrombotic microangiopathy with vacuolated podocytes. GECs are swollen, lose fenestrations, and frequently show sub-endothelial fibrinoid deposits ${ }^{182}$. Podocytes, however, usually are present in normal numbers and have preserved their foot processes. This observation demonstrates that it is possible to disrupt the GFB without disrupting podocytes (FIG. 3a).

Proteomic analysis of patients' plasma samples led to the identification of high circulating levels of a soluble decoy receptor, soluble Fms-like tyrosine kinase 1 (sFLT1; also known as soluble vascular endothelial growth factor receptor 1 (sVEGFR1)), in many patients with pre-eclampsia ${ }^{183,184}$. The abnormally high levels of sFLT1 arise from the placenta weeks before clinical disease ${ }^{183,184}$. The placenta is a rapidly growing highly vascularized organ. It is thought that SFLT1 is an important negative regulator of placental vascular growth and that excessive sFLT1 production results in inadequate new vessel formation, leading to fetal growth retardation. Excessive sFLT1 spills into the maternal circulation and can exert anti-angiogenic activity by binding circulating VEGF, thereby blocking signalling from VEGFR2 (REF. ${ }^{185}$ ). In the kidney, sFLT1 inhibits the activation and signalling of VEGFR2 on GECs by VEGFA from adjacent podocytes. VEGFR2 signalling is critical for GEC maturation, migration into the glomerular tuft, maintenance of fenestration and normal GEC function ${ }^{27,186}$. GEC function in pre-eclampsia can also be affected by reduced levels of pro-angiogenic placental growth factor or increased levels of soluble endoglin, which blocks endoglin-TGF $\beta$ signalling.

Disturbance of these signalling pathways from placenta-derived factors may also have deleterious effects on the $\mathrm{GFB}^{187}$. In support of a critical role for sFLT1 in pre-eclampsia, anti-VEGF antibody therapy, which is used to treat some cancers, can cause GECs to lose fenestrations and function, leading to loss of GFB integrity as an on-target side effect ${ }^{188,189}$. These clinical findings underscore the impact of VEGF sequestration on GEC function, and also highlight the role of cell-cell crosstalk between podocytes and GECs in maintaining the highly differentiated endothelial state that is required for an effective GFB (FIG. 3d).

The mechanisms of the pathological overflow of sFLT1 from the placenta in pre-eclampsia are unknown. However, the ensuing endothelial dysfunction characterized by impaired vasodilation results from a decrease in nitric oxide synthase (NOS) activity and nitric oxide (NO) bioavailability (normally stimulated by VEGF) in the endothelium and increased oxidative stress, which combine to further stimulate systemic hypertension ${ }^{190,191}$. Low levels of endothelial NO therefore likely exacerbate glomerular disease and contribute to proteinuria ${ }^{192-194}$. Degradation of the GEC glycocalyx has also been linked to reduced VEGF signalling by sFLT1 in pre-eclampsia, and may contribute to GFB permeability ${ }^{195-197}$ (FIG. 3d).

GEC dysfunction is the main feature of glomerular involvement in pre-eclampsia. Nevertheless, the reduced expression of podocyte slit diaphragm proteins nephrin and synaptopodin as well as the detection of detached podocytes in the urine are consistent with podocyte injury ${ }^{198}$. Although homeostatic local sFLT1 production has been shown to stabilize podocyte function, excess sFLT1 can also prevent autocrine VEGF from binding to receptors on podocytes, thereby directly affecting the podocyte actin cytoskeleton ${ }^{199,200}$. 
Pre-eclampsia is well managed in the USA with antihypertensive medications, and results in few deaths. Nevertheless, serious complications including liver injury, acute kidney injury, bleeding and seizures are frequent. Clinical studies involving sFLT1 elimination by dextran sulfate apheresis in humans have shown promise in patients with severe disease; apheresis reduces proteinuria and prolongs pregnancy ${ }^{201}$, but does not effectively remove sFLT1. Of note, the therapeutic goal is to reduce sFLT1 levels by $30-40 \%$, as a higher degree of reduction can result in reduced uterine blood flow ${ }^{202,203}$. An academic team from The University of Massachusetts RNA Therapeutics Institute has developed a novel therapeutic approach that blocks the production of sFLT1 by the placenta in rodent and primate models using small interfering RNA (siRNA)-mediated silencing of $s F L T 1$ mRNA. Cholesterol conjugation of siRNAs resulted in stable and sufficient accumulation in rodent and baboon placentae and selectively lowered circulating sFLT1 protein levels by $50 \%{ }^{204}$. This, in turn, alleviated hypertension and proteinuria of pre-eclampsia that was induced in the preclinical models, without adverse consequences to the developing fetus. Therefore, an siRNA-based therapeutic for pre-eclampsia could be highly effective. This therapeutic approach may be taken forward by Tiger Therapeutics.

\section{Enabling technologies}

In addition to the advances in target identification through family studies, genomics, systems biology and proteomics highlighted in the preceding sections, several other breakthroughs have occurred in drug discovery that will enable the advancement of candidate molecules targeting the GFB. With advances in cell isolation and cell culture methods, detection of in situ binding and assays using fluorescent read-outs, it is possible to perform secondary screens of candidate molecules on human patient-derived podocyte monocultures. Such assays may be more relevant than cell lines that have little or no relevance to GFB disease. Still, these monocultures bear only a superficial relationship to podocytes in vivo, and do not recapitulate the complex cellular interplay between GECs and the GBM ${ }^{205,206}$. Protocols for establishing mature podocytes from human induced pluripotent stem cells have been developed ${ }^{207}$, and are now being integrated into more complex model systems such as $3 \mathrm{D}$ cultures and organoids. These emerging technologies can enable low- to medium-throughput evaluation of candidate therapies in model microfluidic systems that contain cells derived from patients. For example, a glomerulus on a chip that hosts GECs and podocytes separated by a porous and flexible poly(dimethylsiloxane) (PDMS) membrane, together with fluidics and strain to better mimic the structure and function of the glomerular capillary wall, can measure the function of the permeability barrier that normally prevents proteinuria ${ }^{208}$. Other developments include a glomerulus on a chip in which endothelial cells and podocytes interact to generate a layer of basal lamina that resembles the GBM, whereas GECs develop a glycocalyx in this system ${ }^{209}$. Exposure of chips to the serum from patients with membranous nephropathy, or use of podocytes derived from a patient with Alport syndrome, showed protein leakage, supporting the chip's potential for use in personalized medicine ${ }^{209}$. These advances allow the evaluation of podocyte function in maintaining the GFB and the assessment of effects of candidates on restoring the GFB in a highly controlled setting ${ }^{208,210}$. Modern homologous recombination and transgenic technologies can be used to place human genes and their regulatory sequences into rodents and test the effects of candidate therapies in vivo against the human target ${ }^{211}$. This has proved important for establishing target engagement and dose in vivo, with the caveat that allometry (modelling dose and pharmacokinetic properties from one species to another) remains a fine art.

In addition to developing better model systems, several additional important advances made over the past decade could enable therapies targeting the GFB to be developed. Structure-based drug design ${ }^{212}$, enabled by the refinement and industrialization of cryogenic electron microscopy ${ }^{213}$, has helped to identify compounds with sub-nanomolar effective inhibitory concentrations. In silico predictive tools have helped tailor arrayed small-molecule screens to focus on classes of compounds, rather than all compounds ${ }^{214}$, and advances have been made in pooled small-molecule screens using DNA-barcoded libraries of small molecules ${ }^{215}$.

The use of in silico tools to predict toxicity, metabolism, production of toxic metabolites or drug-drug interactions has improved the early de-prioritization of molecules with potential toxicity ${ }^{216}$. Screening promising small molecules using standardized assays for potential cardiac toxicity, hepatotoxicity and genotoxicity has become standard practice in the early stages of small-molecule improvement from initial screening hits, but screening against renal toxicity remains challenging. However, human kidney tubule 2D and, particularly, $3 \mathrm{D}$ cultures have shown promise in detecting toxicity in panels of known molecules with nephrotoxicity ${ }^{217}$.

Finally, new therapeutic modalities, including nucleic acid therapies such as ASOs (BOX 1), are proving robust in targeting the GFB. Current algorithms enable the design of ASO molecules that not only engage the target mRNA or microRNA for protracted periods but also appear to adequately engage the target mRNA in the nucleus of podocytes and GECs. Other therapeutic approaches are also being identified that can precisely correct the disease-causing variants and mutations in the GFB. These DNA editors, including CRISPR-Cas9, base editors and prime editors, have the potential to precisely correct the underlying cause of kidney diseases, if they can be delivered to cells of the GFB successfully ${ }^{218,219}$.

\section{Outlook and future directions}

New insights into genetics, defined environmental insults and the structure-function of the GFB have resulted in new candidate therapeutic targets for kidney disease that selectively target the GFB, and offer the possibility of tailored therapies that correct defects within or between cells of the GFB. Modern structure-based small-molecule drug design, advances in nucleic acid therapies and protein therapies have enabled successful drug candidates to emerge that are now in clinical evaluation. The use 
of precision medicine will be required to make these candidate therapies successful in clinical practice.

One of the themes in this Review has been the selection of the right patients to evaluate investigational drugs. An increasing number of trials fail to meet their primary end points, and then a post hoc analysis identifies a subgroup in whom the drug was effective. Development of clinically available biomarkers to identify kidney injury and monitor therapy effectiveness will help guide clinical decisions in the future. For APOL1 inhibitors, their efficacy will likely be seen only in patients who have a diagnosis of FSGS on kidney biopsy and carry high-risk variants on both copies of APOL1. Similarly, an siRNA against sFLT1 will probably only work in patients with pre-eclampsia who also have elevated levels of sFLT1 in the circulation. Kidney disease has long been defined by pathological descriptors on biopsy, but modern genetics and other techniques can increasingly identify patients with a potential genetic or immunological cause, and therapies targeting the GFB are becoming more tailored to those causes.

Although certain rare kidney diseases that alter the GFB are caused by inherited mutations, most adult-onset disease has previously been thought to be acquired. Such conclusions are now being challenged by the finding that common forms of kidney disease, such as hypertensive nephrosclerosis and diabetic kidney disease, occur in patients with heterozygous loss-of-function mutations or high-risk variants that are identical to those that cause rare, early-onset disease ${ }^{47}$. For example, mutations in uromodulin (encoded by $U M O D$ ) and collagen type IV trimers (encoded by COL4A3, COL4A4 and COL4A5) cause autosomal dominant tubulointerstitial kidney disease and Alport syndrome, respectively, but the same mutations or common variants are also detected in patients presenting with FSGS, hypertensive kidney disease and diabetic kidney disease ${ }^{47,220}$. The continued whole-exome sequencing of patients with common forms of kidney disease is likely to refine current targets and identify novel genetically defined therapeutic targets. It is likely that more routine genotyping of patients with kidney disease will determine their eligibility for the next generation of GFB targeted therapies. Nevertheless, improved and validated assays of human disease are required to investigate promising therapeutic targets, enable drug discovery and provide the confidence needed to advance these therapies to the clinic. Current animal models are poor predictors of human disease. Patient-derived 3D cell systems could bridge this translational gap.

Published online 14 July 2021
1. Iseki, K., Ikemiya, Y., Iseki, C. \& Takishita, S. Proteinuria and the risk of developing end-stage renal disease. Kidney Int. 63, 1468-1474 (2003)

2. Fuhrman, D. Y. et al. Albuminuria, proteinuria, and renal disease progression in children with CKD. Clin. J. Am. Soc. Nephrol. 12, 912-920 (2017).

3. Kolb, A. et al. A national registry study of patient and renal survival in adult nephrotic syndrome. Kidney Int. Rep. 6, 449-459 (2021).

4. Abbate, M., Zoja, C. \& Remuzzi, G. How does proteinuria cause progressive renal damage? J. Am. Soc. Nephrol. 17, 2974-2984 (2006).

5. Mann, J. F. E. et al. Liraglutide and renal outcomes in type 2 diabetes. N. Engl. J. Med. 377, 839-848 (2017).

6. Wanner, C. et al. Empagliflozin and progression of kidney disease in type 2 diabetes. N. Engl. J. Med 375, 323-334 (2016).

7. Wang, X. X. et al. SGLT2 protein expression is increased in human diabetic nephropathy: SGLT2 protein inhibition decreases renal lipid accumulation, inflammation, and the development of nephropathy in diabetic mice. J. Biol. Chem. 292, 5335-5348 (2017).

8. Uthman, L. et al. Empagliflozin and dapagliflozin reduce ROS generation and restore NO bioavailability in tumor necrosis factor $\alpha$-stimulated human coronary arterial endothelial cells. Cell. Physiol. Biochem. 53, 865-886 (2019)

9. Cherney, D. Z. I. et al. Effects of the SGLT2 inhibitor dapagliflozin on proteinuria in non-diabetic patients with chronic kidney disease (DIAMOND): a randomised, double-blind, crossover trial. Lancet Diabetes Endocrinol. 8, 582-593 (2020).

10. Levey, A. S. et al. Change in albuminuria and GFR as end points for clinical trials in early stages of CKD: a scientific workshop sponsored by the National Kidney Foundation in collaboration with the US Food and Drug Administration and European Medicines Agency. Am. J. Kidney Dis. 75, 84-104 (2020).

11. Haraldsson, B., Nyström, J. \& Deen, W. M. Properties of the glomerular barrier and mechanisms of proteinuria. Physiol. Rev. 88, 451-487 (2008)

12. Meyrier, A. Focal and segmental glomerulosclerosis: multiple pathways are involved. Semin. Nephrol. 31 326-332 (2011).

13. Pozzi, A. et al. $\beta 1$ integrin expression by podocytes is required to maintain glomerular structural integrity. Dev. Biol. 316, 288-301 (2008).

14. Faul, C., Asanuma, K., Yanagida-Asanuma, E., Kim, K. $\&$ Mundel, P. Actin up: regulation of podocyte structure and function by components of the actin cytoskeleton. Trends Cell Biol. 17, 428-437 (2007).

15. Perico, L., Conti, S., Benigni, A. \& Remuzzi, G. Podocyte-actin dynamics in health and disease. Nat. Rev. Nephrol. 12, 692-710 (2016).

16. Kestila, M. et al. Positionally cloned gene for a novel glomerular protein - nephrin - is mutated in congenital nephrotic syndrome. Mol. Cell 1, 575-582 (1998).

17. Boute, N. et al. NPHS2, encoding the glomerular protein podocin, is mutated in autosomal recessive steroid-resistant nephrotic syndrome. Nat. Genet. 24 349-354 (2000); erratum 25, 125 (2000).

18. Grahammer, F. et al. A flexible, multilayered protein scaffold maintains the slit in between glomerular podocytes. JCl Insight 1, e86177 (2016).

19. Winn, M. P. et al. A mutation in the TRPC 6 cation channel causes familial focal segmental glomerulosclerosis. Science 308, 1801-1804 (2005).

20. Reiser, J. et al. TRPC6 is a glomerular slit diaphragmassociated channel required for normal renal function. Nat. Genet. 37, 739-744 (2005).

21. Yao, J. et al. a-Actinin-4-mediated FSCS: an inherited kidney disease caused by an aggregated and rapidly degraded cytoskeletal protein. PLOS Biol. 2, e167 (2004).

22. Gigante, M. et al. CD2AP mutations are associated with sporadic nephrotic syndrome and focal segmental glomerulosclerosis (FSCS). Nephrol. Dial. Transpl. 24, 1858-1864 (2009).

23. Brown, E. J et al. Mutations in the formin gene INF2 cause focal segmental glomerulosclerosis. Nat. Genet 42, 72-76 (2010).

24. Ronco, P. Proteinuria: is it all in the foot? J. Clin. Invest. 117, 2079-2082 (2007).

25. Campanholle, G., Ligresti, G., Gharib, S. A. \& Duffield, J. S. Cellular mechanisms of tissue fibrosis. 3 Novel mechanisms of kidney fibrosis. Am. J. Physiol. Cell Physiol. 304, C591-C603 (2013).

26. Butt, L. et al. A molecular mechanism explaining albuminuria in kidney disease. Nat. Metab. 2 461-474 (2020)

27. Sison, K. et al. Glomerular structure and function require paracrine, not autocrine, VEGF-VEGFR-2 signaling. J. Am. Soc. Nephrol. 21, 1691-1701 (2010)

28. Jeansson, M. et al. Angiopoietin-1 is essential in mouse vasculature during development and in response to injury. J. Clin. Invest. 121, 2278-2289 (2011).

29. Ballermann, B. J. Contribution of the endothelium to the glomerular permselectivity barrier in health and disease. Nephron Physiol. 106, p19-p25 (2007).
30. Fogo, A. B. \& Kon, V. The glomerulus - a view from the inside - the endothelial cell. Int. J. Biochem. Cell Biol. 42, 1388-1397 (2010).

31. Haraldsson, B. \& Nystrom, J. The glomerular endothelium: new insights on function and structure. Curr. Opin. Nephrol. Hypertens. 21, 258-263 (2012).

32. Jeansson, M., Bjorck, K., Tenstad, O. \& Haraldsson, B. Adriamycin alters glomerular endothelium to induce proteinuria. J. Am. Soc. Nephrol. 20, 114-122 (2009).

33. Singh, A. et al. High glucose causes dysfunction of the human glomerular endothelial glycocalyx. Am. J. Physiol. Ren. Physiol. 300, F40-F48 (2011).

34. Sun, Y. B. et al. Glomerular endothelial cell injury and damage precedes that of podocytes in adriamycin-induced nephropathy. PLOS ONE 8 , e55027 (2013).

35. Caprioli, J. et al. Genetics of HUS: the impact of MCP, $\mathrm{CFH}$, and IF mutations on clinical presentation, response to treatment, and outcome. Blood 108 1267-1279 (2006)

36. Zhao, H. J. et al. Endothelial nitric oxide synthase deficiency produces accelerated nephropathy in diabetic mice. J. Am. Soc. Nephrol. 17, 2664-2669 (2006).

37. Yuen, D. A. et al. eNOS deficiency predisposes podocytes to injury in diabetes. J. Am. Soc. Nephrol. 23, 1810-1823 (2012)

38. Satchell, S. C. \& Tooke, J. E. What is the mechanism of microalbuminuria in diabetes: a role for the glomerular endothelium? Diabetologia 51, 714-725 (2008).

39. Lajer, M. et al. Plasma concentration of asymmetric dimethylarginine (ADMA) predicts cardiovascular morbidity and mortality in type 1 diabetic patients with diabetic nephropathy. Diabetes Care 31 747-752 (2008).

40. Hanai, K. et al. Asymmetric dimethylarginine is closely associated with the development and progression of nephropathy in patients with type 2 diabetes. Nephrol. Dial. Transpl. 24, 1884-1888 (2009).

41. Shibata, R. et al. Involvement of asymmetric dimethylarginine (ADMA) in tubulointerstitial ischaemia in the early phase of diabetic nephropathy. Nephrol. Dial. Transpl. 24, 1162-1169 (2009).

42. Dellamea, B. S., Pinto, L. C., Leitao, C. B., Santos, K. C. \& Canani, L. H. Endothelial nitric oxide synthase gene polymorphisms and risk of diabetic nephropathy: a systematic review and meta-analysis. BMC Med. Genet. 15, 9 (2014). 
43. Zanchi, A. et al. Risk of advanced diabetic nephropathy in type 1 diabetes is associated with endothelial nitric oxide synthase gene polymorphism. Kidney Int. 57, 405-413 (2000).

44. Wang, Y. et al. COL4A3 gene variants and diabetic kidney disease in MODY. Clin. J. Am. Soc. Nephrol. 13 , 1162-1171 (2018)

45. Salem, R. M. et al. Genome-wide association study of diabetic kidney disease highlights biology involved in glomerular basement membrane collagen. J. Am. Soc Nephrol. 30, 2000-2016 (2019).

46. Sadowski, C. E. et al. A single-gene cause in $29.5 \%$ of cases of steroid-resistant nephrotic syndrome J. Am. Soc. Nephrol. 26, 1279-1289 (2015).

47. Groopman, E., Goldstein, D. \& Gharavi, A. Diagnostic utility of exome sequencing for kidney disease. N. Engl. J. Med. 380, 2080-2081 (2019).

48. Mele, C. et al. MYO1E mutations and childhood familial focal segmental glomerulosclerosis. N. Engl. J. Med. 365, 295-306 (2011).

49. Schuler, M. H. et al. Miro1-mediated mitochondrial positioning shapes intracellular energy gradients required for cell migration. Mol. Biol. Cell 28 2159-2169 (2017)

50. Tang, C. et al. Mitochondrial quality control in kidney injury and repair. Nat. Rev. Nephrol. 17, 299-318 (2020).

51. Daniel, R., Mengeta, A., Bilodeau, P. \& Lee, J. M Mitochondria tether to focal adhesions during cell migration and regulate their size. Preprint at bioRxiv https://doi.org/10.1101/827998 (2019).

52. Brinkkoetter, P. T. et al. Anaerobic glycolysis maintains the glomerular filtration barrier independent of mitochondrial metabolism and dynamics. Cell Rep 27, 1551-1566.e5 (2019).

53. Baek, J. H. et al. Deletion of the mitochondrial complex-IV cofactor heme a:farnesyltransferase causes focal segmental glomerulosclerosis and interferon response. Am. J. Pathol. 188, 2745-2762 (2018).

54. Lowik, M. M., Hol, F. A., Steenbergen, E. J. Wetzels, J. F. \& van den Heuvel, L. P. Mitochondrial tRNALeu(UUR) mutation in a patient with steroidresistant nephrotic syndrome and focal segmental glomerulosclerosis. Nephrol. Dial. Transpl. 20, 336-341 (2005)

55. Guery, B. et al. The spectrum of systemic involvement in adults presenting with renal lesion and mitochondrial tRNA(Leu) gene mutation. J. Am. Soc Nephrol. 14, 2099-2108 (2003).

56. Casalena, G. et al. Mpv17 in mitochondria protects podocytes against mitochondrial dysfunction and apoptosis in vivo and in vitro. Am. J. Physiol. Ren. Physiol. 306, F1372-F1380 (2014).

57. Widmeier, E. et al. ADCK4 deficiency destabilizes the coenzyme $\mathrm{Q}$ complex, which is rescued by 2,4-dihydroxybenzoic acid treatment. J. Am. Soc Nephrol. 31, 1191-1211 (2020).

58. Herlitz, L. C. et al. Tenofovir nephrotoxicity: acute tubular necrosis with distinctive clinical, pathological, and mitochondrial abnormalities. Kidney Int. 78, 1171-1177 (2010)

59. Ayanga, B. A. et al. Dynamin-related protein deficiency improves mitochondrial fitness and protects against progression of diabetic nephropathy. J. Am. Soc. Nephrol. 27, 2733-2747 (2016).

60. Kawakami, T. et al. Deficient autophagy results in mitochondrial dysfunction and FSGS. J. Am. Soc. Nephrol. 26, 1040-1052 (2015).

61. Daehn, I. et al. Endothelial mitochondrial oxidative stress determines podocyte depletion in segmental glomerulosclerosis. J. Clin. Invest. 124, 1608-1621 (2014).

62. Qi, H. et al. Glomerular endothelial mitochondrial dysfunction is essential and characteristic of diabetic kidney disease susceptibility. Diabetes 66, 763-778 (2017).

63. Fu, J. et al. Comparison of glomerular and podocyte mRNA profiles in streptozotocin-induced diabetes. J. Am. Soc. Nephrol. 27, 1006-1014 (2016)

64. Ebefors, K. et al. Endothelin receptor-A mediates degradation of the glomerular endothelial surface layer via pathologic crosstalk between activated podocytes and glomerular endothelial cells. Kidney Int. 96, 957-970 (2019).

65. Baigent, C. \& Lennon, R. Should we increase GFR with bardoxolone in Alport syndrome? J. Am. Soc. Nephrol. 29, 357-359 (2018)

66. Dinkova-Kostova, A. T. \& Abramov, A. Y. The emerging role of Nrf2 in mitochondrial function. Free Radic. Bio Med. 88, 179-188 (2015)

67. Scarpulla, R. C. Metabolic control of mitochondrial biogenesis through the PGC-1 family regulatory network. Biochim. Biophys. Acta 1813, 1269-1278 (2011).

68. Gomez, I. G. et al. Anti-microRNA-21 oligonucleotides prevent Alport nephropathy progression by stimulating metabolic pathways. J. Clin. Invest. 125 $141-156$ (2015)

69. Ducasa, G. M. et al. ATP-binding cassette A deficiency causes cardiolipin-driven mitochondrial dysfunction in podocytes. J. Clin. Invest. 129 3387-3400 (2019).

70. Casalena, G. A. et al. The diabetic microenvironment causes mitochondrial oxidative stress in glomerular endothelial cells and pathological crosstalk with podocytes. Cell Commun. Signal. 18, 105 (2020).

71. Ma, L. et al. APOL1 renal-risk variants induce mitochondrial dysfunction. J. Am. Soc. Nephrol. 28 1093-1105 (2017).

72. Kopp, J. B. et al. MYH9 is a major-effect risk gene for focal segmental glomerulosclerosis. Nat. Genet. $\mathbf{4 0}$ 1175-1184 (2008)

73. Genovese, G. et al. Association of trypanolytic ApoL variants with kidney disease in African Americans. Science 329, 841-845 (2010).

74. Larsen, C. P., Beggs, M. L., Saeed, M. \& Walker, P. D. Apolipoprotein L1 risk variants associate with systemic lupus erythematosus-associated collapsing glomerulopathy. J. Am. Soc. Nephrol. 24, 722-725 (2013).

75. Duran, C. E. et al. Prevalence of APOL1 risk variants in afro-descendant patients with chronic kidney disease in a Latin American country. Int. J. Nephrol. 2019, 7076326 (2019).

76. Riella, C. et al. APOL1-associated kidney disease in Brazil. Kidney Int. Rep. 4, 923-929 (2019).

77. Friedman, D. J., Kozlitina, J., Genovese, G., Jog, P. \& Pollak, M. R. Population-based risk assessment of APOL1 on renal disease. J. Am. Soc. Nephrol. 22 , 2098-2105 (2011)

78. Dummer, P. D. et al. APOL1 kidney disease risk variants: an evolving landscape. Semin. Nephrol. 35 222-236 (2015)

79. Nichols, B. et al Innate immunity pathways regulate the nephropathy gene apolipoprotein L1. Kidney Int 87, 332-342 (2015)

80. Wu, H. et al. AKI and collapsing glomerulopathy associated with COVID-19 and APOL 1 high-risk genotype. J. Am. Soc. Nephrol. 31, 1688-1695 (2020).

81. Vanwalleghem, G. et al. Coupling of lysosomal and mitochondrial membrane permeabilization in trypanolysis by APOL1. Nat. Commun. 6, 8078 (2015).

82. Bruno, J., Pozzi, N., Oliva, J. \& Edwards, J. C. Apolipoprotein $\mathrm{L} 1$ confers $\mathrm{pH}$-switchable ion permeability to phospholipid vesicles. J. Biol. Chem 292, 18344-18353 (2017)

83. Kruzel-Davila, E. et al. APOL1-mediated cell injury involves disruption of conserved trafficking processes. J. Am. Soc. Nephrol. 28, 1117-1130 (2017).

84. Bruggeman, L. A., O'Toole, J. F. \& Sedor, J. R. APOL1 polymorphisms and kidney disease: loss-of-function or gain-of-function? Am. J. Physiol. Ren. Physiol. 316 , F1-F8 (2019).

85. Lee, B. T. et al. The APOL1 genotype of African American kidney transplant recipients does not impact 5-year allograft survival. Am. J. Transplant. 12 1924-1928 (2012).

86. Freedman, B. I. et al. APOL1 genotype and kidney transplantation outcomes from deceased African American donors. Transplantation 100, 194-202 (2016).

87. Beckerman, P. et al. Transgenic expression of human APOL1 risk variants in podocytes induces kidney disease in mice. Nat. Med. 23, 429-438 (2017)

88. Khatua, A. K. et al. Exon 4-encoded sequence is a major determinant of cytotoxicity of apolipoprotein L1. Am. J. Physiol. Cell Physiol. 309, C22-C37 (2015).

89. Chun, J et al. Recruitment of APOL 1 kidney disease risk variants to lipid droplets attenuates cell toxicity. Proc. Natl Acad. Sci. USA 116, 3712-3721 (2019).

90. Shah, S. S. et al. APOL1 kidney risk variants induce cell death via mitochondrial translocation and opening of the mitochondrial permeability transition pore. J. Am. Soc. Nephrol. 30, 2355-2368 (2019).

91. Olabisi, O. A. et al. APOL1 kidney disease risk variants cause cytotoxicity by depleting cellular potassium and inducing stress-activated protein kinases. Proc. Natl Acad. Sci. USA 113, 830-837 (2016).

92. Fu, Y. et al. APOL1-G1 in nephrocytes induces hypertrophy and accelerates cell death. J. Am. Soc. Nephrol. 28, 1106-1116 (2017).
93. Wen, $\mathrm{H}$. et al. APOL1 risk variants cause podocytes injury through enhancing endoplasmic reticulum stress. Biosci. Rep. 38, BSR20171713 (2018).

94. Okamoto, K. et al. APOL1 risk allele RNA contributes to renal toxicity by activating protein kinase $\mathrm{R}$. Commun. Biol. 1, 188 (2018).

95. Aghajan, M. et al. Antisense oligonucleotide treatment ameliorates IFN- $\gamma$-induced proteinuria in APOL 1-transgenic mice. JCl Insight 4, e126124 (2019).

96. Liang, S. S. et al. Clinico-pathological characteristics and outcomes of patients with biopsy-proven hypertensive nephrosclerosis: a retrospective cohort study. BMC Nephrol. 17, 42 (2016).

97. Vanhollebeke, B. et al. Human Trypanosoma evansi infection linked to a lack of apolipoprotein L-I. N. Engl. J. Med. 355, 2752-2756 (2006).

98. Kormann, R. et al. Roles of APOL1 G1 and G2 variants in sickle cell disease patients: kidney is the main target. Br. J. Haematol. 179, 323-335 (2017).

99. Freedman, B I et al. End-stage renal disease in African Americans with lupus nephritis is associated with APOL1. Arthritis Rheumatol. 66, 390-396 (2014).

100. Quinzii, C. et al. A mutation in para-hydroxybenzoate polyprenyl transferase (COQ2) causes primary coenzyme $\mathrm{Q} 10$ deficiency. Am. J. Hum. Genet. 78, 345-349 (2006).

101. Heeringa, S. F. et al. CoQ6 mutations in human patients produce nephrotic syndrome with sensorineural deafness. J. Clin. Invest. 121, 2013-2024 (2011).

102. Lopez, L. C. et al. Leigh syndrome with nephropathy and $\mathrm{CoO}_{10}$ deficiency due to decaprenyl diphosphate synthase subunit 2 (PDSS2) mutations. Am. J. Hum. Genet. 79, 1125-1129 (2006).

103. Ashraf, S. et al. ADCK4 mutations promote steroid-resistant nephrotic syndrome through $\mathrm{CoO}_{10}$ biosynthesis disruption. J. Clin. Invest. 123, 5179-5189 (2013).

104. Korkmaz, E. et al. ADCK4-associated glomerulopathy causes adolescence-onset FSGS. J. Am. Soc. Nephrol. 27, 63-68 (2016).

105. Widmeier, E. et al. Treatment with 2,4-dihydroxybenzoic acid prevents FSCS progression and renal fibrosis in podocyte-specific Coq6 knockout mice. J. Am. Soc. Nephrol. 30, 393-405 (2019).

106. Stanczyk, M., Balasz-Chmielewska, I., LipskaZietkiewicz, B. \& Tkaczyk, M. CoO 10 -related sustained remission of proteinuria in a child with $\mathrm{COQ} 6$ glomerulopathy - a case report. Pediatr. Nephrol. 33, 2383-2387 (2018).

107. Feng, $C$. et al. Coenzyme $Q_{10}$ supplementation therapy for 2 children with proteinuria renal disease and ADCK4 mutation: case reports and literature review. Medicine 96, e8880 (2017)

108. Pierrel, F. Impact of chemical analogs of 4-hydroxybenzoic acid on coenzyme $\mathrm{Q}$ biosynthesis: from inhibition to bypass of coenzyme $Q$ deficiency. Front. Physiol. 8, 436 (2017).

109. Doimo, M. et al. Effect of vanillic acid on $\mathrm{COQ} 6$ mutants identified in patients with coenzyme Q10 deficiency. Biochim. Biophys. Acta 1842, 1-6 (2014).

110. Freyer, C. et al. Rescue of primary ubiquinone deficiency due to a novel $\mathrm{COQ} 7$ defect using 2,4-dihydroxybensoic acid. J. Med. Genet. 52 779-783 (2015).

111. Sidhom, E. H. et al. Targeting a Braf/Mapk pathway rescues podocyte lipid peroxidation in $\mathrm{CoO}$ deficiency kidney disease. J. Clin. Invest. 131, e141380 (2021).

112. Gee H Y et al. ARHGDIA mutations cause nephrotic syndrome via defective RHO GTPase signaling. J. Clin. Invest. 123, 3243-3253 (2013)

113. Akilesh, S. et al. Arhgap24 inactivates Rac1 in mouse podocytes, and a mutant form is associated with familial focal segmental glomerulosclerosis. J. Clin. Invest 121, 4127-4137 (2011).

114. Subramanian, B. et al. Mice with mutant Inf2 show impaired podocyte and slit diaphragm integrity in response to protamine-induced kidney injury. Kidney Int. 90, 363-372 (2016).

115. Subramanian, B. et al. FSGS-causing INF2 mutation impairs cleaved INF2 N-fragment functions in podocytes. J. Am. Soc. Nephrol. 31, 374-391 (2020)

116. Schell, C. et al. ARP3 controls the podocyte architecture at the kidney filtration barrier. Dev. Cell 47, 741-357.e8 (2018)

117. Sever, S., Damke, H. \& Schmid, S. L. Dynamin: GTP controls the formation of constricted coated pits, the rate limiting step in clathrin-mediated endocytosis. J. Cell Biol. 150, 1137-1148 (2000). 
118. Gu, C. et al. Direct dynamin-actin interactions regulate the actin cytoskeleton. $E M B O \mathrm{~J} .29$ 3593-3606 (2010).

119. Schiffer, M. et al. Pharmacological targeting of actindependent dynamin oligomerization ameliorates chronic kidney disease in diverse animal models. Nat. Med. 21, 601-609 (2015).

120. Hill, T. et al. Small molecule inhibitors of dynamin I GTPase activity: development of dimeric tyrphostins J. Med. Chem. 48, 7781-7788 (2005)

121. Greka, A. \& Mundel, P. Calcium regulates podocyte actin dynamics. Semin. Nephrol. 32, 319-326 (2012).

122. Riehle, M. et al. TRPC6 G757D loss-of-function mutation associates with FSGS. J. Am. Soc. Nephrol. 27, 2771-2783 (2016)

123. Chiluiza, D., Krishna, S., Schumacher, V. A. \& Schlondorff, J. Gain-of-function mutations in transient receptor potential C6 (TRPC6) activate extracellular signal-regulated kinases $1 / 2$ (ERK1/2). J. Biol. Chem. 288, 18407-18420 (2013).

124. Kuwahara, K. et al. TRPC6 fulfills a calcineurin signaling circuit during pathologic cardiac remodeling. J. Clin. Invest. 116, 3114-3126 (2006).

125. Schlondorff, J., Del Camino, D., Carrasquillo, R., Lacey, V. \& Pollak, M. R. TRPC6 mutations associated with focal segmental glomerulosclerosis cause constitutive activation of NFAT-dependent transcription. Am. J. Physiol. Cell Physiol. 296 , C558-C569 (2009).

126. Wang, Y. et al. Activation of NFAT signaling in podocytes causes glomerulosclerosis. J. Am. Soc. Nephrol. 21, 1657-1666 (2010).

127. Farmer, L. K. et al. TRPC6 binds to and activates calpain, independent of its channel activity, and regulates podocyte cytoskeleton, cell adhesion, and motility. J. Am. Soc. Nephrol. 30, 1910-1924 (2019).

128. Krall, P. et al. Podocyte-specific overexpression of wild type or mutant Trpc6 in mice is sufficient to cause glomerular disease. PLoS ONE 5, e1 2859 (2010)

129. Eckel, J. et al. TRPC6 enhances angiotensin II-induced albuminuria. J. Am. Soc. Nephrol. 22, 526-535 (2011).

130. Bai, Y. et al. Structural basis for pharmacological modulation of the TRPC6 channel. eLife 9, e53311 (2020).

131. Tian, D. Q. et al. Antagonistic regulation of actin dynamics and cell motility by TRPC5 and TRPC 6 channels. Sci. Signal. 3, ra77 (2010).

132. Wang, X. X. et al. TRPC5 does not cause or aggravate glomerular disease. J. Am. Soc. Nephrol. 29, 409-415 (2018)

133. Schaldecker, T. et al. Inhibition of the TRPC5 ion channel protects the kidney filter. J. Clin. Invest. 123 , 5298-5309 (2013)

134. Zhou, Y. et al. A small-molecule inhibitor of TRPC5 ion channels suppresses progressive kidney disease in animal models. Science 358, 1332-1336 (2017).

135. Yu, H. et al. Rac1 activation in podocytes induces rapid foot process effacement and proteinuria. Mol. Cell Biol. 33, 4755-4764 (2013).

136. Wei, C. L. et al. Circulating urokinase receptor as a cause of focal segmental glomerulosclerosis. Nat. Med. 17, 952-960 (2011).

137. Wei, C. et al. UPAR isoform 2 forms a dimer and induces severe kidney disease in mice. J. Clin. Invest. 129, 1946-1959 (2019)

138. Tzima, E., del Pozo, M. A., Shattil, S. J., Chien, S. \& Schwartz, M. A. Activation of integrins in endothelia cells by fluid shear stress mediates Rho-dependent cytoskeletal alignment. EMBO J. 20, 4639-4647 (2001).

139. Wei, C. et al. Circulating suPAR in two cohorts of primary FSCS. J. Am. Soc. Nephrol. 23, 2051-2059 (2012).

140. Hayek, S. S. et al. Soluble urokinase receptor and chronic kidney disease. N. Engl. J. Med. 373. 1916-1925 (2015)

141. Botha, S. et al. Soluble urokinase plasminogen activator receptor as a prognostic marker of all-cause and cardiovascular mortality in a black population. Int J Cardiol 184, 631-636 (2015)

142. Bock, M. E., Price, H. E., Gallon, L. \& Langman, C. B. Serum soluble urokinase-type plasminogen activator receptor levels and idiopathic FSGS in children: a single-center report. Clin. J. Am. Soc. Nephrol. 8, 1304-1311 (2013)

143. Franco Palacios, C. R. et al. Urine but not serum soluble urokinase receptor (suPAR) may identify cases of recurrent FSGS in kidney transplant candidates. Transplantation 96, 394-399 (2013).
144. Spinale, J. M. et al. A reassessment of soluble urokinase-type plasminogen activator receptor in glomerular disease. Kidney Int. 87, 564-574 (2015).

145. Walden Biosciences Launches. Walden Biosciences https://www.waldenbiosciences.com/waldenbiosciences-launches-to-transform-the-treatmentof-kidney-disease/ (2020).

146. Wang, H. et al. Noninvasive assessment of antenatal hydronephrosis in mice reveals a critical role for Robo 2 in maintaining anti-reflux mechanism. PLoS ONE 6, e24763 (2011).

147. Hwang, D. Y. et al. Mutations of the SLIT2-ROBO2 pathway genes SLIT2 and SRGAP1 confer risk for congenital anomalies of the kidney and urinary tract. Hum. Genet. 134, 905-916 (2015).

148. Lu, W. N. et al. Disruption of ROBO2 is associated with urinary tract anomalies and confers risk of vesicoureteral reflux. Am. J. Kidney Dis. 49, A56-A56 (2007).

149. Fan, X. P. et al. Inhibitory effects of Robo2 on nephrin: a crosstalk between positive and negative signals regulating podocyte structure. Cell Rep. 2, 52-61 (2012).

150. Fan, X. P. et al. SLIT2/ROBO2 signaling pathway inhibits nonmuscle myosin IIA activity and destabilizes kidney podocyte adhesion. JCl Insight 1, e86934 (2016).

151. Kopp, J. B. Glomerular pathology in autosomal dominant MYH9 spectrum disorders: what are the clues telling us about disease mechanism? Kidney Int. 78, 130-133 (2010)

152. Johnstone, D. B. et al. Podocyte-specific deletion of Myh9 encoding nonmuscle myosin heavy chain 2A predisposes mice to glomerulopathy. Mol. Cell. Biol. 31, 2162-2170 (2011)

153. Pisarek-Horowitz, A. et al. Loss of roundabout guidance receptor 2 (Robo2) in podocytes protects adult mice from glomerular injury by maintaining podocyte foot process structure. Am. J. Pathol. 190 799-816 (2020)

154. Sakurai, T., Yanagisawa, M. \& Masaki, T. Molecular characterization of endothelin receptors. Trends Pharmacol. Sci. 13, 103-108 (1992).

155. Zeravica, R. et al. Plasma endothelin- 1 levels and albuminuria in patients with type 2 diabetes mellitus. Med. Pregl. 69, 140-145 (2016).

156. Zanatta, C. M. et al. Endothelin-1 levels and albuminuria in patients with type 2 diabetes mellitus. Diabetes Res. Clin. Pract. 80, 299-304 (2008).

157. Chen, H. C. et al. Plasma and urinary endothelin-1 in focal segmental glomerulosclerosis. J. Clin. Lab. Anal. 15, 59-63 (2001).

158. Gupta, R. M. et al. A genetic variant associated with five vascular diseases is a distal regulator of endothelin-1 gene expression. Cell 170, 522-533. e15 (2017).

159. Rahman, T., Baker, M., Hall, D. H., Avery, P. J. \& Keavney, B. Common genetic variation in the type A endothelin-1 receptor is associated with ambulatory blood pressure: a family study. J. Hum. Hypertens. 22 , 282-288 (2008)

160. Sorensen, S. S., Madsen, J. K. \& Pedersen, E. B. Systemic and renal effect of intravenous infusion of endothelin-1 in healthy human volunteers. Am. J. Physiol. 266, F411-F418 (1994)

161. Badr, K. F. et al. Mesangial cell, glomerular and renal vascular responses to endothelin in the rat kidney. Elucidation of signal transduction pathways. J. Clin. Invest 83, 336-342 (1989).

162. Shin, S. J., Lee, Y. J. \& Tsai, J. H. The correlation of plasma and urine endothelin-1 with the severity of nephropathy in Chinese patients with type 2 diabetes. Scand. J. Clin. Lab. Invest. 56, 571-576 (1996).

163. Collino, F. et al. Preeclamptic sera induce nephrin shedding from podocytes through endothelin-1 release by endothelial glomerular cells. Am. J. Physiol Ren. Physiol. 294, F1185-F1194 (2008).

164. Saleh, M. A., Boesen, E. I., Pollock, J. S., Savin, V. J. \& Pollock, D. M. Endothelin-1 increases glomerular permeability and inflammation independent of blood pressure in the rat. Hypertension 56, 942-949 (2010).

165. Miao, L., Dai, Y. \& Zhang, J. Mechanism of RhoA/Rho kinase activation in endothelin-1-induced contraction in rabbit basilar artery. Am. J. Physiol. Heart Circ. Physiol. 283, H983-H989 (2002).

166. Clerk, A. et al. Regulation of mitogen-activated protein kinases in cardiac myocytes through the small G protein Rac1. Mol. Cell. Biol. 21, 1173-1184 (2001).

167. Morigi, M. et al. In response to protein load podocytes reorganize cytoskeleton and modulate endothelin-1 gene: implication for permselective dysfunction of chronic nephropathies. Am. J. Pathol. 166, 1309-1320 (2005)

168. Morigi, M. et al. Shigatoxin-induced endothelin-1 expression in cultured podocytes autocrinally mediates actin remodeling. Am. J. Pathol. 169 1965-1975 (2006).

169. Barton, M. Therapeutic potential of endothelin receptor antagonists for chronic proteinuric renal disease in humans. Biochim. Biophys. Acta 1802 1203-1213 (2010).

170. Barton, M. \& Tharaux, P. L. Endothelin and the podocyte. Clin. Kidney J. 5, 17-27 (2012).

171. Lenoir, O. et al. Direct action of endothelin-1 on podocytes promotes diabetic glomerulosclerosis. J. Am. Soc. Nephrol. 25, 1050-1062 (2014).

172. Lee, T. M., Chung, T. H., Lin, S. Z. \& Chang, N. C. Endothelin receptor blockade ameliorates renal injury by inhibition of RhoA/Rho-kinase signalling in deoxycorticosterone acetate-salt hypertensive rats. J. Hypertens. 32, 795-805 (2014).

173. Callera, G. E. \& Bendhack, L. M. Mechanisms underlying the contractile response to endothelin-1 in the rat renal artery. Pharmacology 68, 131-139 (2003).

174. Nina A. Van de Lest, M. Z., Wolterbeek, R., Bruijn, J. A \& Scharpfenecker, M. Altered podocyte-endothelial cross-talk and increased oxidative stress in patients with FSGS [published abstract]. Kidney Week ASN TH-PO1059 (2019).

175. Boels, M. G. et al. Atrasentan reduces albuminuria by restoring the glomerular endothelial glycocalyx barrier in diabetic nephropathy. Diabetes 65, 2429-2439 (2016).

176. Garsen, M. et al. Endothelin-1 induces proteinuria by heparanase-mediated disruption of the glomerular glycocalyx. J. Am. Soc. Nephrol. 27, 3545-3551 (2016).

177. Barton, M. Endothelin antagonism and reversal of proteinuric renal disease in humans. Contrib. Nephrol. 172, 210-222 (2011).

178. Kohan, D. E. Endothelin, hypertension and chronic kidney disease: new insights. Curr. Opin. Nephrol. Hypertens. 19, 134-139 (2010)

179. Heerspink, H. J. L. et al. Atrasentan and renal events in patients with type 2 diabetes and chronic kidney disease (SONAR): a double-blind, randomised, placebo-controlled trial. Lancet 393, 1937-1947 (2019).

180. Trachtman, H. et al. DUET: a phase 2 study evaluating the efficacy and safety of sparsentan in patients with FSGS. J. Am. Soc. Nephrol. 29, 2745-2754 (2018).

181. Calizo, R. C. et al. Disruption of podocyte cytoskeletal biomechanics by dasatinib leads to nephrotoxicity. Nat. Commun. 10, 2061 (2019).

182. Stillman, I. E. \& Karumanchi, S. A. The glomerular injury of preeclampsia. J. Am. Soc. Nephrol. 18, 2281-2284 (2007).

183. Maynard, S. E. et al. Excess placental soluble fms-like tyrosine kinase 1 (sFlt1) may contribute to endothelial dysfunction, hypertension, and proteinuria in preeclampsia. J. Clin. Invest. 111, 649-658 (2003).

184. Levine, R. J. et al. Circulating angiogenic factors and the risk of preeclampsia. N. Engl. J. Med. 350, 672-683 (2004).

185. Alon, T. et al. Vascular endothelial growth-factor acts as a survival factor for newly formed retinal-vessels and has implications for retinopathy of prematurity. Nat. Med. 1, 1024-1028 (1995).

186. Ferrara, N. Role of vascular endothelial growth factor in the regulation of angiogenesis. Kidney Int. $\mathbf{5 6}$ 794-814 (1999).

187. Muller-Deile, J. et al. Overexpression of preeclampsia induced microRNA-26a-5p leads to proteinuria in zebrafish. Sci. Rep. 8, 3621 (2018).

188. Eremina, V. et al. VEGF inhibition and renal thrombotic microangiopathy. N. Engl. J. Med. 358, 1129-1136 (2008).

189. Sugimoto, H. et al. Neutralization of circulating vascular endothelial growth factor (VEGF) by anti-VEGF antibodies and soluble VEGF receptor 1 (sFIt-1) induces proteinuria. J. Biol. Chem. 278, 12605-12608 (2003).

190. Matsubara, K., Higaki, T., Matsubara, Y. \& Nawa, A Nitric oxide and reactive oxygen species in the pathogenesis of preeclampsia. Int. J. Mol. Sci. 16, 4600-4614 (2015).

191. Venkatesha, S. et al. Soluble endoglin contributes to the pathogenesis of preeclampsia. Nat. Med. 12 642-649 (2006).

192. Li, F. et al. eNOS deficiency acts through endothelin to aggravate sFIt-1-induced pre-eclampsia-like phenotype. J. Am. Soc. Nephrol. 23, 652-660 (2012). 
193. Serrano, N. C. et al. Endothelial NO synthase genotype and risk of preeclampsia - a multicenter case-control study. Hypertension 44, 702-707 (2004).

194. Fatini, C. et al. Endothelial nitric oxide synthase gene influences the risk of pre-eclampsia, the recurrence of negative pregnancy events, and the maternal-fetal flow. J. Hypertens. 24, 1823-1829 (2006).

195. Weissgerber, T. L. et al. Early onset preeclampsia is associated with glycocalyx degradation and reduced microvascular perfusion. J. Am. Heart Assoc. 8 e010647 (2019).

196. Reitsma, S., Slaaf, D. W., Vink, H., van Zandvoort, M. A \& oude Egbrink, M. G. The endothelial glycocalyx: composition, functions, and visualization. Pflug. Arch. 454, 345-359 (2007).

197. Butler, M. J., Down, C. J., Foster, R. R. \& Satchell, S. C The pathological relevance of increased endothelial glycocalyx permeability. Am. J. Pathol. 190, 742-751 (2020).

198. Garovic, V. D. et al. Urinary podocyte excretion as a marker for preeclampsia. Am. J. Obstet. Gynecol. 196, 320-327 (2007).

199. Jin, J. et al. Soluble FLT1 binds lipid microdomains in podocytes to control cell morphology and glomerular barrier function. Cell 151, 384-399 (2012).

200. Bertuccio, C., Veron, D., Aggarwal, P. K., Holzman, L. $\&$ Tufro, A. Vascular endothelial growth factor receptor 2 direct interaction with nephrin links VEGF-A signals to actin in kidney podocytes. J. Biol. Chem 286, 39933-39944 (2011).

201. Thadhani, R. et al. Removal of soluble fms-like tyrosine kinase- 1 by dextran sulfate apheresis in preeclampsia. J. Am. Soc. Nephrol. 27, 903-913 (2016).

202. Thadhani, R. et al. Pilot study of extracorporeal removal of soluble fms-like tyrosine kinase 1 in preeclampsia. Circulation 124, 940-950 (2011).

203. Khankin, E. V., Mandala, M., Colton, I., Karumanchi, S. A. \& Osol, G. Hemodynamic, vascular, and reproductive impact of FMS-like tyrosine kinase 1 (FLT1) blockade on the uteroplacental circulation during normal mouse pregnancy. Biol. Reprod. $\mathbf{8 6}$ 57 (2012).

204. Turanov, A. A. et al. RNAi modulation of placental SFLT1 for the treatment of preeclampsia. Nat. Biotechnol. 36, 1164-1173 (2018).

205. Chadli, L. et al. Identification of regulators of the myofibroblast phenotype of primary dermal fibroblasts from early diffuse systemic sclerosis patients. Sci. Rep. 9, 4521 (2019).

206. Ebefors, K., Lassēn, E., Anandakrishnan, N., Azeloglu, E. U. \& Daehn, I. S. Modeling the glomerular filtration barrier and intercellular crosstalk. Front Physiol. 12, 772 (2021)

207. Musah, S., Dimitrakakis, N., Camacho, D. M Church, G. M. \& Ingber, D. E. Directed differentiation of human induced pluripotent stem cells into mature kidney podocytes and establishment of a Glomerulus Chip. Nat. Protoc. 13, 1662-1685 (2018).

208. Musah, S. et al. Mature induced-pluripotent-stem-cellderived human podocytes reconstitute kidney glomerular-capillary-wall function on a chip. Nat. Biomed. Eng. 1, 0069 (2017)

209. Petrosyan, A. et al. A glomerulus-on-a-chip to recapitulate the human glomerular filtration barrier Nat. Commun. 10, 3656 (2019).

210. Dang, L. T. H. et al. Hyperactive FOXO1 results in lack of tip stalk identity and deficient microvascular regeneration during kidney injury. Biomaterials 141 . 314-329 (2017)

211. Peltz, G. Can 'humanized' mice improve drug development in the 21 st century? Trends Pharmacol. Sci. 34, 255-260 (2013).

212. de Ruyck, J., Brysbaert, G., Blossey, R. \& Lensink, M. F. Molecular docking as a popular tool in drug design, an in silico travel. Adv. Appl. Bioinform. Chem. 9, 1-1 (2016).

213. Su, Q. et al. Structure of the human PKD1-PKD2 complex. Science 361, eaat9819 (2018).

214. Kuhlman, B. \& Bradley, P. Advances in protein structure prediction and design. Nat. Rev. Mol. Cell Biol. 20, 681-697 (2019).

215. Goodnow, R. A., Dumelin, C. E. \& Keefe, A. D. DNA-encoded chemistry: enabling the deeper sampling of chemical space. Nat. Rev. Drug Discov. 16, 131-147 (2017)

216. Lehmann, D. F., Eggleston, W. D. \& Wang, D. Validation and clinical utility of the $\mathrm{hERG} I \mathrm{IC}_{50}: \mathrm{C}_{\max }$ ratio to determine the risk of drug-induced torsades de pointes: a meta-analysis. Pharmacotherapy 38 , 341-348 (2018)

217. Weber, E. J. et al. Human kidney on a chip assessment of polymyxin antibiotic nephrotoxicity. JCl Insight 3 . e123673 (2018)

218. Anzalone, A. V. et al. Search-and-replace genome editing without double-strand breaks or donor DNA. Nature 576, 149-157 (2019).

219. Porto, E. M., Komor, A. C., Slaymaker, I. M. \& Yeo, G. W. Base editing: advances and therapeutic opportunities. Nat. Rev. Drug Discov. 19, 839-859 (2020).

220. Kottgen, A. et al. Multiple loci associated with indices of renal function and chronic kidney disease. Nat. Genet. 41, 712-717 (2009).
221. Pedigo, C. E. et al. Local TNF causes NFATc1dependent cholesterol-mediated podocyte injury. J. Clin. Invest. 126, 3336-3350 (2016).

222. Komers, R. et al. Study design of the phase 3 sparsentan versus irbesartan (DUPLEX) study in patients with focal segmental glomerulosclerosis. Kidney Int. Rep. 5, 494-502 (2020).

223. Lassén, E. ¿ Daehn, I. S. Molecular mechanismsin early diabetic kidney disease: glomerular endothelial cell dysfunction. Int. J. Mol. Sci. 21, 9456 (2020).

224. Dias, N. \& Stein, C. A. Antisense oligonucleotides: basic concepts and mechanisms. Mol. Cancer Ther. 1 , 347-355 (2002).

225. Hung, G. N. et al. Characterization of target mRNA reduction through in situ RNA hybridization in multiple organ systems following systemic antisense treatment in animals. Nucleic Acid Ther. 23, 369-378 (2013).

226. Engelhardt, J. A. Comparative renal toxicopathology of antisense oligonucleotides. Nucleic Acid Ther. 26 199-209 (2016)

227. Crooke, S. T. et al. The effects of 2'-O-methoxyethyl oligonucleotides on renal function in humans. Nucleic Acid Ther. 28, 10-22 (2018).

\section{Acknowledgements}

I.S.D. is supported by National Institutes of Health (NIH) grant R01 DK097253 and Department of Defense CDMRP grant E01 W81XWH2010836. Illustrations used during submission were created using BioRender.

\section{Author contributions}

The authors contributed equally to all aspects of the article.

\section{Competing interests}

J.S.D. is a former employee of Vertex Pharmaceuticals, which is developing VX-147 for the treatment of APOL1-mediated kidney disease, holds Vertex stock and holds patents for the invention and use of Lademirsen for the treatment of kidney diseases including Alport syndrome. I.S.D. declares no competing interests.

\section{Peer review information}

Nature Reviews Drug Discovery thanks A. Fornoni and the other, anonymous, reviewers for their contribution to the peer review of this work.

Publisher's note

Springer Nature remains neutral with regard to jurisdictional claims in published maps and institutional affiliations.

C) Springer Nature Limited 2021 